\title{
A General Multidimensional Monte Carlo Approach for Dynamic Hedging under Stochastic Volatility
}

\author{
Daniel Bonetti, ${ }^{1}$ Dorival Leão, ${ }^{2}$ Alberto Ohashi, ${ }^{3}$ and Vinícius Siqueira ${ }^{2}$ \\ ${ }^{1}$ Departamento de Sistemas de Computacção, Universidade de São Paulo, 13560-970 São Carlos, SP, Brazil \\ ${ }^{2}$ Departamento de Matemática Aplicada e Estatística, Universidade de São Paulo, 13560-970 São Carlos, SP, Brazil \\ ${ }^{3}$ Departamento de Matemática, Universidade Federal da Paraíba, 13560-970 João Pessoa, PB, Brazil
}

Correspondence should be addressed to Alberto Ohashi; alberto.ohashi@pq.cnpq.br

Received 22 August 2014; Accepted 14 December 2014

Academic Editor: Enzo Orsingher

Copyright (C) 2015 Daniel Bonetti et al. This is an open access article distributed under the Creative Commons Attribution License, which permits unrestricted use, distribution, and reproduction in any medium, provided the original work is properly cited.

\begin{abstract}
We propose a feasible and constructive methodology which allows us to compute pure hedging strategies with respect to arbitrary square-integrable claims in incomplete markets. In contrast to previous works based on PDE and BSDE methods, the main merit of our approach is the flexibility of quadratic hedging in full generality without a priori smoothness assumptions on the payoff. In particular, the methodology can be applied to multidimensional quadratic hedging-type strategies for fully path-dependent options with stochastic volatility and discontinuous payoffs. In order to demonstrate that our methodology is indeed applicable, we provide a Monte Carlo study on generalized Föllmer-Schweizer decompositions, locally risk minimizing, and mean variance hedging strategies for vanilla and path-dependent options written on local volatility and stochastic volatility models.
\end{abstract}

\section{Introduction}

1.1. Background and Motivation. Let $(S, \mathbf{F}, \mathbb{P})$ be a financial market composed by a continuous F-semimartingale $S$ which represents a discounted risky asset price process, $\mathbf{F}=\left\{\mathbf{F}_{t} ; 0 \leq\right.$ $t \leq T\}$ is a filtration which encodes the information flow in the market on a finite horizon $[0, T], \mathbb{P}$ is a physical probability measure, and $\mathscr{M}^{e}$ is the set of equivalent local martingale measures. Let $H$ be an $\mathbf{F}_{T}$-measurable contingent claim describing the net payoff whose trader is faced at time $T$. In order to hedge this claim, the trader has to choose a dynamic portfolio strategy.

Under the assumption of an arbitrage-free market, the classical Galtchouk-Kunita-Watanabe (henceforth abbreviated as GKW) decomposition yields

$$
H=\mathbb{E}_{\mathbb{Q}}[H]+\int_{0}^{T} \theta_{\ell}^{H, \mathbb{Q}} d S_{\ell}+L_{T}^{H, \mathbb{Q}} \quad \text { under } \mathbb{Q} \in \mathscr{M}^{e}
$$

where $L^{H, Q}$ is a $\mathbb{Q}$-local martingale which is strongly orthogonal to $S$ and $\theta^{H, Q}$ is an adapted process.
The GKW decomposition plays a crucial role in determining optimal hedging strategies in a general Brownianbased market model subject to stochastic volatility $\sigma$. For instance, if $S$ is a one-dimensional Itô risky asset price process which is adapted to the information generated by a twodimensional Brownian motion $W=\left(W^{(1)}, W^{(2)}\right)$, then there exists a two-dimensional adapted process $\phi^{H, \mathbb{Q}}:=$ $\left(\phi^{H, 1}, \phi^{H, 2}\right)$ such that

$$
H=\mathbb{E}_{\mathbb{Q}}[H]+\int_{0}^{T} \phi_{t}^{H, \mathbb{Q}} d W_{t}
$$

which also realizes

$$
\theta_{t}^{H, \mathbb{Q}}=\phi_{t}^{H, 1}\left[S_{t} \sigma_{t}\right]^{-1}, \quad L_{t}^{H, \mathbb{Q}}=\int_{0}^{t} \phi^{H, 2} d W_{s}^{(2)} ; \quad 0 \leq t \leq T .
$$

In the complete market case, there exists a unique $\mathbb{Q} \in$ $\mathscr{M}^{e}$ and, in this case, $L^{H, \mathbb{Q}}=0, \mathbb{E}_{\mathbb{Q}}[H]$, is the unique fair price and the hedging replicating strategy is fully described by the process $\theta^{H, Q}$. In a general stochastic volatility framework, there are infinitely many GKW orthogonal decompositions 
parameterized by the set $\mathscr{M}^{e}$ and hence one can ask if it is possible to determine the notion of non-self-financing optimal hedging strategies solely based on the quantities (3). This type of question was firstly answered by Föllmer and Sondermann [1] and later on extended by Schweizer [2] and Föllmer and Schweizer [3] through the existence of the socalled Föllmer-Schweizer decomposition which turns out to be equivalent to the existence of locally risk minimizing hedging strategies. The GKW decomposition under the socalled minimal martingale measure constitutes the starting point to get locally risk minimizing strategies provided that one is able to check some square-integrability properties of the components in (1) under the physical measure. See, for example, $[4,5]$ for details and other references therein. See also, for example, [6], where Fölmer-Schweizer decompositions can be retrieved by solving linear backward stochastic differential equations (BSDEs). Orthogonal decompositions without square-integrability properties can also be defined in terms of the the so-called generalized Föllmer-Schweizer decomposition (see, e.g., [7]).

In contrast to the local risk minimization approach, one can insist on working with self-financing hedging strategies which give rise to the so-called mean variance hedging methodology. In this approach, the spirit is to minimize the expectation of the squared hedging error over all initial endowments $x$ and all suitable admissible strategies $\varphi \in \Theta$ :

$$
\inf _{\varphi \in \Theta, x \in \mathbb{R}} \mathbb{E}_{\mathbb{P}}\left|H-x-\int_{0}^{T} \varphi_{t} d S_{t}\right|^{2} .
$$

The nature of the optimization problem (4) suggests to work with the subset $\mathscr{M}_{2}^{e}:=\left\{\mathbb{Q} \in \mathscr{M}^{e} ; d \mathbb{Q} / d \mathbb{P} \in L^{2}(\mathbb{P})\right\}$. Rheinlander and Schweizer [9], Gourieroux et al. [10], and Schweizer [11] show that if $\mathscr{M}_{2}^{e} \neq \emptyset$ and $H \in L^{2}(\mathbb{P})$, then the optimal quadratic hedging strategy exists and it is given by $\left(\mathbb{E}_{\widetilde{\mathbb{P}}}[H], \eta^{\widetilde{\mathbb{P}}}\right)$, where

$$
\begin{array}{r}
\eta_{t}^{\widetilde{\mathbb{P}}}:=\theta_{t}^{H, \widetilde{\mathbb{P}}}-\frac{\widetilde{\zeta}_{t}}{\widetilde{Z}_{t}}\left(V_{t-}^{H, \widetilde{\mathbb{P}}}-\mathbb{E}_{\widetilde{\mathbb{P}}}[H]-\int_{0}^{t} \eta_{\ell}^{\widetilde{\mathbb{P}}} d S_{\ell}\right) ; \\
0 \leq t \leq T .
\end{array}
$$

Here $\theta^{H, \widetilde{\mathbb{P}}}$ is computed in terms of $\widetilde{\mathbb{P}}$; the so-called variance optimal martingale measure, $\tilde{\zeta}$, realizes

$$
\widetilde{Z}_{t}:=\mathbb{E}_{\widetilde{\mathbb{P}}}\left[\frac{d \widetilde{\mathbb{P}}}{d \mathbb{P}} \mid \mathbf{F}_{t}\right]=\widetilde{Z}_{0}+\int_{0}^{t} \widetilde{\zeta}_{\ell} d S_{\ell} ; \quad 0 \leq t \leq T,
$$

and $V .^{H, \widetilde{\mathbb{P}}}:=\mathbb{E}_{\widetilde{\mathbb{P}}}[H \mid \mathbf{F}$. $]$ is the value option price process under $\widetilde{\mathbb{P}}$. See also Černý and Kallsen [12] for the general semimartingale case and the works [13-15] for other utilitybased hedging strategies based on GKW decompositions.

Concrete representations for the pure hedging strategies $\left\{\theta^{H, \mathbb{Q}} ; \mathbb{Q}=\widehat{\mathbb{P}}, \widetilde{\mathbb{P}}\right\}$ can in principle be obtained by computing cross-quadratic variations $d\left[V^{H, \mathbb{Q}}, S\right]_{t} / d[S, S]_{t}$ for $\mathbb{Q} \in$ $\{\widetilde{\mathbb{P}}, \widehat{\mathbb{P}}\}$. For instance, in the classical vanilla case, pure hedging strategies can be computed by means of the Feynman-Kac theorem (see, e.g., [4]). In the path-dependent case, the obtention of concrete computationally efficient representations for $\theta^{H, \mathbb{Q}}$ is a rather difficult problem. Feynman-Kactype arguments for fully path-dependent options mixed with stochastic volatility typically face not-well-posed problems on the whole trading period; highly degenerate PDEs arise in this context as well. Generically speaking, one has to work with non-Markovian versions of the Feynman-Kac theorem in order to get robust dynamic hedging strategies for fully path-dependent options written on stochastic volatility risky asset price processes.

In the mean variance case, the only quantity in (5) not related to GKW decomposition is $\widetilde{Z}$ which can in principle be expressed in terms of the so-called fundamental representation equations given by Hobson [16] and Biagini et al. [17] in the stochastic volatility case. For instance, Hobson derives closed form expressions for $\widetilde{\zeta}$ and also for any type of $q$-optimal measure in the Heston model [18]. Recently, semiexplicit formulas for vanilla options based on general characterizations of the variance-optimal hedge in Cerný and Kallsen [12] have been also proposed in the literature which allow for a feasible numerical implementation in affine models. See Kallsen and Vierthauer [19] and Černý and Kallsen [20] for some results in this direction.

A different approach based on linear BSDEs can also be used in order to get useful characterizations for the optimal hedging strategies. In this case, concrete numerical schemes for BSDEs play a key role in applications. In the Markovian case, there are several efficient methods. See, for example, Delong [6] and other references therein. In the non-Markovian case, when the terminal value is allowed to depend on the whole history of a forward diffusion, the difficulty is notorious. One fundamental issue is the implementation of feasible approximations for the "martingale integrand" of BSDEs. To the best of our knowledge, all the existing numerical methods require a priori regularity conditions on the final condition. See, for example, $[6,21-$ 23] and other references therein. Recently, Briand and Labart [24] use Malliavin calculus methods to compute conditional expectations based on Wiener chaos expansions under some regularity conditions. See also the recent results announced by Gobet and Turkedjiev $[25,26]$ by using regression methods.

1.2. Contribution of the Current Paper. The main contribution of this paper is the obtention of flexible and computationally efficient multidimensional non-Markovian representations for generic option price processes which allow for a concrete computation of the associated GKW decomposition $\left(\theta^{H, \mathbb{Q}}, L^{H, \mathbb{Q}}\right)$ for $\mathbb{Q}$-square-integrable payoffs $H$ with $\mathbb{Q} \in \mathscr{M}^{e}$. We provide a Monte Carlo methodology able to compute optimal quadratic hedging strategies with respect to general square-integrable claims in a multidimensional Brownianbased market model. In contrast to previous works (see, e.g., [6] and other references therein), the main contribution of this paper is the formulation of a concrete numerical scheme for quadratic hedging (local risk minimization) under full generality, where only square-integrability assumption is 
imposed. As far as the mean variance hedging is concerned, we are able to compute pure optimal hedging strategies $\theta^{H, \widetilde{\mathbb{P}}}$ for arbitrary square-integrable payoffs. Hence, our methodology also applies to this case provided that one is able to compute the fundamental representation equations in Hobson [16] and Biagini et al. [17] which is the case for the classical Heston model.

The starting point of this paper is based on weak approximations developed by Leão and Ohashi [27] for one-dimensional Brownian functionals. They introduced a one-dimensional space-filtration discretization scheme constructed from suitable waiting times which measure the instants when the Brownian motion hits some a priori levels. In the present work, we extend [27] in one direction: we provide a feasible numerical scheme for multidimensional $\mathbb{Q}$-GKW decompositions under rather weak integrability conditions for a given $\mathbb{Q} \in \mathscr{M}^{e}$. In order to apply our methodology for hedging, we analyze the convergence of our approximating hedging strategies to the respective value processes in a Brownian-based incomplete market setup. This allows us to perform quadratic hedging for generic squareintegrable payoffs written on stochastic volatility models. The numerical scheme of this work can also be viewed as part of a more general theory concerning a weak version of functional Itô calculus (see $[28,29]$ ) as introduced by Ohashi et al. [30]. We implement the multidimensional weak derivative operators defined in [30] in the pure martingale case to solve hedging problems in generic stochastic volatility models.

In this paper, the multidimensional numerical scheme for martingale representations lies in the exact simulation of an i.i.d sequence of increments of hitting times

$$
T_{n}^{k, j}:=\inf \left\{t>T_{n-1}^{k, j}:\left|W_{t}^{(j)}-W_{T_{n-1}^{k, j}}^{(j)}\right|=\epsilon_{k}\right\} ; \quad n \geq 1,
$$

where $T_{0}^{k, j}:=0$ for $1 \leq j \leq p$ and $\epsilon_{k} \downarrow 0$ as $k \rightarrow \infty$. The fundamental object which allows us to obtain a numerical scheme for $\theta^{H, \mathbb{Q}}$ is the following ratio:

$$
\mathbb{E}_{\mathbb{Q}}\left[\frac{\mathbb{E}_{\mathbb{Q}}\left[H \mid \mathscr{F}_{T_{1}^{k, j}}^{k}\right]-\mathbb{E}_{\mathbb{Q}}\left[H \mid \mathscr{F}_{\tau_{1}^{k, j}}^{k}\right]}{W_{T_{1}^{k, j}}^{(j)}}\right], \quad j=1, \ldots, d,
$$

for $d \leq p$, where $\tau_{1}^{k, j}:=\max \left\{T_{n}^{k} ; T_{n}^{k}<T_{1}^{k, j}\right\}, T_{0}^{k}:=0$, and

$$
T_{n}^{k}:=\inf _{\substack{1 \leq j \leq p \\ m \geq 1}}\left\{T_{m}^{k, j} ; T_{m}^{k, j} \geq T_{n-1}^{k}\right\},
$$

for $n \geq 1$. Here, there are $d$ asset price processes driven by a $p$-dimensional Brownian motion $\left(W^{(1)}, \ldots, W^{(p)}\right)$. By approximating the payoff $H$ in terms of functionals of the random walks

$$
A_{t}^{k, j}:=W_{T_{n}^{k, j}}^{(j)} \quad \text { on }\left\{T_{n}^{k, j} \leq t<T_{n+1}^{k, j}\right\}
$$

we will take advantage of the discrete structure of the sigmaalgebras in (37) to evaluate (8) by standard Monte Carlo methods. The information set contained in $\left(\mathscr{F}_{T_{1}^{k, j}}^{k}, \mathscr{F}_{\tau_{1}^{k, j}}^{k}\right)$ is perfectly implementable by using the algorithm proposed by Burq and Jones [8]. We leave the implementation of simulation-regression method for a further study.

In order to demonstrate that our methodology is indeed applicable, we provide a Monte Carlo study on generalized Föllmer-Schweizer decompositions, locally risk minimizing and mean variance hedging strategies for vanilla and pathdependent options written on local volatility and stochastic volatility models. The numerical experiments suggest that pure hedging strategies based on generalized FöllmerSchweizer decompositions mitigate very well the cost of hedging of a path-dependent option even if there is no guarantee of the existence of locally risk minimizing strategies. We also compare hedging errors arising from optimal mean variance hedging strategies for one-touch options written on a Heston model with nonzero correlation.

Lastly, we want to emphasize the fact that it is our chief goal is to provide a feasible numerical method which works in full generality. In this case, the price we pay is to work with weak convergence results instead of $L^{p}$ or uniform convergence in probability. We leave a more refined analysis on error estimates and rates of convergence under Markovian assumptions to a future research.

The remainder of this paper is structured as follows. In Section 2, we fix the notation and we describe the basic underlying market model. In Section 3, we provide the basic elements of the Monte Carlo methodology proposed in this paper. In Section 4, we formulate dynamic hedging strategies starting from a given GKW decomposition and we translate our results to well-known quadratic hedging strategies. The Monte Carlo algorithm and the numerical study are described in Sections 5 and 6, respectively. The Appendix presents more refined approximations when the martingale representations admit additional hypotheses.

\section{Preliminaries}

Throughout this paper, we assume that we are in the usual Brownian market model with finite time horizon $0<$ $T<\infty$ equipped with the stochastic basis $(\Omega, \mathbf{F}, \mathbb{P})$ generated by a standard $p$-dimensional Brownian motion $B=\left\{\left(B_{t}^{(1)}, \ldots, B_{t}^{(p)}\right) ; 0 \leq t<\infty\right\}$ starting from 0 . The filtration $\mathbf{F}:=\left(\mathbf{F}_{t}\right)_{0 \leq t \leq T}$ is the $\mathbb{P}$-augmentation of the natural filtration generated by $B$. For a given $m$-dimensional vector $J=\left(J_{1}, \ldots, J_{m}\right)$, we denote by $\operatorname{diag}(J)$ the $m \times m$ diagonal matrix whose $\ell$ th diagonal term is $J_{\ell}$. In this paper, for all unexplained terminology concerning general theory of processes, we refer to Dellacherie and Meyer [31].

In view of stochastic volatility models, let us split $B$ into two multidimensional Brownian motions as follows: $B^{S}:=$ $\left(B^{(1)}, \ldots, B^{(d)}\right)$ and $B^{I}:=\left(B^{(d+1)}, \ldots, B^{(p)}\right)$. In this section, the market consists of $d+1$ assets $(d \leq p)$ : one riskless asset given by

$$
d S_{t}^{0}=r_{t} S_{t}^{0} d t, \quad S_{0}^{0}=1 ; \quad 0 \leq t \leq T,
$$


and a $d$-dimensional vector of risky assets $\bar{S}:=\left(\bar{S}^{1}, \ldots, \bar{S}^{d}\right)$ which satisfies the following stochastic differential equation:

$$
\begin{aligned}
d \bar{S}_{t}=\operatorname{diag}\left(\bar{S}_{t}\right)\left(b_{t} d t+\sigma_{t} d B_{t}^{S}\right), & \bar{S}_{0}=\bar{x} \in \mathbb{R}^{d} ; \\
0 & \leq t \leq T .
\end{aligned}
$$

Here, the real-valued interest rate process $r=\left\{r_{t} ; 0 \leq\right.$ $t \leq T\}$, the vector of mean rates of return $b:=\left\{b_{t}=\right.$ $\left.\left(b_{t}^{1}, \ldots, b_{t}^{d}\right) ; 0 \leq t \leq T\right\}$, and the volatility matrix $\sigma:=$ $\left\{\sigma_{t}=\left(\sigma_{t}^{i j}\right) ; 1 \leq i \leq d, 1 \leq j \leq d, 0 \leq t \leq T\right\}$ are assumed to be predictable and they satisfy the standard assumptions in such way that both $S^{0}$ and $\bar{S}$ are well-defined positive semimartingales. We also assume that the volatility matrix $\sigma$ is nonsingular for almost all $(t, \omega) \in[0, T] \times \Omega$. The discounted price $S:=\left\{S_{i}:=\bar{S}^{i} / S^{0} ; i=1, \ldots, d\right\}$ follows

$$
\begin{array}{r}
d S_{t}=\operatorname{diag}\left(S_{t}\right)\left[\left(b_{t}-r_{t} \mathbf{1}_{d}\right) d t+\sigma_{t} d B_{t}^{S}\right] ; \quad S_{0}=x \in \mathbb{R}^{d}, \\
0 \leq t \leq T,
\end{array}
$$

where $\mathbf{1}_{d}$ is a $d$-dimensional vector with every component equal to 1 . The market price of risk is given by

$$
\psi_{t}:=\sigma_{t}^{-1}\left[b_{t}-r_{t} \mathbf{1}_{d}\right], \quad 0 \leq t \leq T,
$$

where we assume

$$
\int_{0}^{T}\left\|\psi_{u}\right\|_{\mathbb{R}^{d}}^{2} d u<\infty \quad \text { a.s. }
$$

In the sequel, $\mathscr{M}^{e}$ denotes the set of $\mathbb{P}$-equivalent probability measures $\mathbb{Q}$ such that, respectively, Radon-Nikodym derivative process is a $\mathbb{P}$-martingale and the discounted price $S$ is a $\mathbb{Q}$-local martingale. Throughout this paper, we assume that $\mathscr{M}^{e} \neq \emptyset$. In our setup, it is well known that $\mathscr{M}^{e}$ is given by the subset of probability measures with Radon-Nikodym derivatives of the form

$$
\begin{aligned}
\frac{d \mathbb{Q}}{d \mathbb{P}}:=\exp [ & -\int_{0}^{T} \psi_{u} d B_{u}^{S}-\int_{0}^{T} \nu_{u} d B_{u}^{I} \\
& \left.-\frac{1}{2} \int_{0}^{T}\left\{\left\|\psi_{u}\right\|_{\mathbb{R}^{d}}^{2}+\left\|\nu_{u}\right\|_{\mathbb{R}^{p-d}}^{2}\right\} d u\right],
\end{aligned}
$$

for some $\mathbb{R}^{p-d}$-valued adapted process $v$ such that $\int_{0}^{T}\left\|v_{t}\right\|_{\mathbb{R}^{p-d}}^{2} d t<\infty$ a.s.

Example 1. The typical example studied in the literature is the following one-dimensional stochastic volatility model:

$$
\begin{aligned}
& d S_{t}=S_{t} \mu\left(t, S_{t}, \sigma_{t}\right) d t+S_{t} \sigma_{t} d Y_{t}^{(1)}, \\
& d \sigma_{t}^{2}=a\left(t, S_{t}, \sigma_{t}\right) d t+b\left(t, S_{t}, \sigma_{t}\right) d Y_{t}^{(2)} ; \quad 0 \leq t \leq T,
\end{aligned}
$$

where $Y^{(1)}$ and $Y^{(2)}$ are correlated Brownian motions with correlation $\rho \in[-1,1]$ and $\mu, a$, and $b$ are suitable functions such that $\left(S, \sigma^{2}\right)$ is a well-defined two-dimensional
Markov process. All continuous stochastic volatility models commonly used in practice fit into specification (17). In this case, $p=2>d=1$ and we recall that the market is incomplete where the set $\mathscr{M}^{e}$ is infinity. The dynamic hedging procedure turns out to be quite challenging due to extrinsic randomness generated by the nontradeable volatility, specially with respect to to exotic options.

2.1. GKW Decomposition. In the sequel, we take $\mathbb{Q} \in \mathscr{M}^{e}$ and we set $W^{S}:=\left(W^{(1)}, \ldots, W^{(d)}\right)$ and $W^{I}:=\left(W^{(d+1)}, \ldots, W^{(p)}\right)$, where

$$
W_{t}^{(j)}:= \begin{cases}B_{t}^{(j)}+\int_{0}^{t} \psi_{u}^{j} d u, & j=1, \ldots, d \\ B_{t}^{(j)}+\int_{0}^{t} v_{u}^{j} d u, & j=d+1, \ldots, p ; 0 \leq t \leq T,\end{cases}
$$

is a standard $p$-dimensional Brownian motion under the measure $\mathbb{Q}$ and filtration $\mathbb{F}:=\left\{\mathscr{F}_{t} ; 0 \leq t \leq T\right\}$ generated by $W=\left(W^{(1)}, \ldots, W^{(p)}\right)$. In what follows, we fix a discounted contingent claim $H$. Recall that the filtration $\mathbb{F}$ is contained in F, but it is not necessarily equal. In the remainder of this paper, we assume the following hypothesis.

(M) The contingent claim $H$ is also $\mathscr{F}_{T}$-measurable.

Remark 2. Assumption (M) is essential for the approach taken in this work because the whole algorithm is based on the information generated by the Brownian motion $W$ (defined under the measure $\mathbb{Q}$ and filtration $\mathbb{F}$ ). As long as the short rate is deterministic, this hypothesis is satisfied for any stochastic volatility model of form (17) and a payoff $\Phi\left(S_{t} ; 0 \leq\right.$ $t \leq T$ ) where $\Phi: \mathscr{C}_{T} \rightarrow \mathbb{R}$ is a Borel map and $\mathscr{C}_{T}$ is the usual space of continuous paths on $[0, T]$. Hence, $(M)$ holds for a very large class of examples founded in practice.

For a given $\mathbb{Q}$-square-integrable claim $H$, the Brownian martingale representation (computed in terms of $(\mathbb{F}, \mathbb{Q})$ ) yields

$$
H=\mathbb{E}_{\mathbb{Q}}[H]+\int_{0}^{T} \phi_{u}^{H, \mathbb{Q}} d W_{u},
$$

where $\phi^{H, \mathbb{Q}}:=\left(\phi^{H, \mathbb{Q}, 1}, \ldots, \phi^{H, \mathbb{Q}, p}\right)$ is a $p$-dimensional $\mathbb{F}$ predictable process. In what follows, we set $\phi^{H, \mathbb{Q}, S}:=\left(\phi^{H, \mathbb{Q}, 1}\right.$, $\left.\ldots, \phi^{H, \mathbb{Q}, d}\right), \phi^{H, \mathbb{Q}, I}:=\left(\phi^{H, \mathbb{Q}, d+1}, \ldots, \phi^{H, \mathbb{Q}, p}\right)$, and

$$
\begin{array}{r}
L_{t}^{H, \mathbb{Q}}:=\int_{0}^{t} \phi_{u}^{H, \mathbb{Q}, I} d W_{u}^{I}, \quad \widehat{V}_{t}:=\mathbb{E}_{\mathbb{Q}}\left[H \mid \mathscr{F}_{t}\right] ; \\
0 \leq t \leq T .
\end{array}
$$

The discounted stock price process has the following $\mathbb{Q}$ dynamics:

$$
d S_{t}=\operatorname{diag}\left(S_{t}\right) \sigma_{t} d W_{t}^{S}, \quad S_{0}=x, \quad 0 \leq t \leq T,
$$


and therefore the $\mathbb{Q}$-GKW decomposition for the pair of locally square-integrable local martingales $(\widehat{V}, S)$ is given by

$$
\begin{aligned}
\widehat{V}_{t} & =\mathbb{E}_{\mathbb{Q}}[H]+\int_{0}^{t} \phi_{u}^{H, \mathbb{Q}, S} d W_{u}^{S}+L_{t}^{H, \mathbb{Q}} \\
& =\mathbb{E}_{\mathbb{Q}}[H]+\int_{0}^{t} \theta_{u}^{H, \mathbb{Q}} d S_{u}+L_{t}^{H, \mathbb{Q}} ; \quad 0 \leq t \leq T,
\end{aligned}
$$

where

$$
\theta^{H, \mathbb{Q}}:=\phi^{H, \mathbb{Q}, S}[\operatorname{diag}(S) \sigma]^{-1}
$$

The $p$-dimensional process $\phi^{H, \mathbb{Q}}$ which constitutes $(20)$ and (23) plays a major role in several types of hedging strategies in incomplete markets and it will be our main object of study.

Remark 3. If we set $v^{j}=0$ for $j=d+1, \ldots, p$ and the correspondent density process is a martingale, then the resulting minimal martingale measure $\widehat{\mathbb{P}}$ yields a GKW decomposition where $L^{H, \widehat{\mathbb{P}}}$ is still a $\mathbb{P}$-local martingale orthogonal to the martingale component of $S$ under $\mathbb{P}$. In this case, it is also natural to implement a pure hedging strategy based on $\theta^{H, \widehat{\mathbb{P}}}$ regardless of the existence of the Föllmer-Schweizer decomposition. If this is the case, this hedging strategy can be based on the generalized Föllmer-Schweizer decomposition (see, e.g., Th. 9 in [7]).

\section{The Random Skeleton and Weak Approximations for GKW Decompositions}

In this section, we provide the fundamentals of the numerical algorithm of this paper for the obtention of hedging strategies in complete and incomplete markets.

3.1. The Multidimensional Random Skeleton. At first, we fix once and for all $\mathbb{Q} \in \mathscr{M}^{e}$ and a $\mathbb{Q}$-square-integrable contingent claim $H$ satisfying $(\mathrm{M})$. In the remainder of this section, we are going to fix a $\mathbb{Q}$-Brownian motion $W$ and with a slight abuse of notation all $\mathbb{Q}$-expectations will be denoted by $\mathbb{E}$. The choice of $\mathbb{Q} \in \mathscr{M}^{e}$ is dictated by the pricing and hedging method used by the trader.

In the sequel, $[\cdot, \cdot]$ denotes the usual quadratic variation between semimartingales and the usual jump of a process is denoted by $\Delta Y_{t}=Y_{t}-Y_{t-}$ where $Y_{t-}$ is the left-hand limit of a cadlag process $Y$. For a pair $(a, b) \in \mathbb{R}^{2}$, we denote $a \vee$ $b:=\max \{a, b\}$ and $a \wedge b:=\min \{a, b\}$. Moreover, for any two stopping times $S$ and $J$, we denote the stochastic intervals $\llbracket S, J \llbracket:=\{(\omega, t) ; S(\omega) \leq t<J(\omega)\}, \llbracket S \rrbracket:=\{(\omega, t) ; S(\omega)=t\}$ and so on. Throughout this article, Leb denotes the Lebesgue measure on the interval $[0, T]$.

For a fixed positive integer $k$ and for each $j=1,2, \ldots, p$ we define $T_{0}^{k, j}:=0$ a.s. and

$$
\begin{array}{r}
T_{n}^{k, j}:=\inf \left\{T_{n-1}^{k, j}<t<\infty ;\left|W_{t}^{(j)}-W_{T_{n-1}^{k, j}}^{(j)}\right|=2^{-k}\right\}, \\
n \geq 1,
\end{array}
$$

where $W:=\left(W^{(1)}, \ldots, W^{(p)}\right)$ is the $p$-dimensional $\mathbb{Q}$ Brownian motion as defined in (18).

For each $j \in\{1, \ldots, p\}$, the family $\mathscr{T}^{k, j}:=\left\{T_{n}^{k, j} ; n \geq 0\right\}$ is a sequence of $\mathbb{F}$-stopping times where the increments $\left\{T_{n}^{k, j}\right.$ $\left.T_{n-1}^{k, j} ; n \geq 1\right\}$ are an i.i.d sequence with the same distribution as $T_{1}^{k, j}$. In the sequel, we define $A^{k}:=\left(A^{k, 1}, \ldots, A^{k, p}\right)$ as the $p$ dimensional step process given in a component-wise manner by

$$
A_{t}^{k, j}:=\sum_{n=1}^{\infty} 2^{-k} \eta_{n}^{k, j} \mathbb{1}_{\left\{T_{n}^{k, j} \leq t\right\}} ; \quad 0 \leq t \leq T
$$

where

$$
\eta_{n}^{k, j}:= \begin{cases}1 ; & \text { if } W_{T_{n}^{k, j}}^{(j)}-W_{T_{n-1}^{k, j}}^{(j)}=2^{-k}, T_{n}^{k, j}<\infty \\ -1 ; & \text { if } W_{T_{n}^{k, j}}^{(j)}-W_{T_{n-1}^{k, j}}^{(j)}=-2^{-k}, T_{n}^{k, j}<\infty \\ 0 ; & \text { if } T_{n}^{k, j}=\infty\end{cases}
$$

for $k, n \geq 1$, and $j=1, \ldots, p$. We split $A^{k}$ into $\left(A^{S, k}, A^{I, k}\right)$ where $A^{S, k}$ is the $d$-dimensional process constituted by the first $d$ components of $A^{k}$ and $A^{I, k}$ and the remainder of the $p-d$-dimensional process. Let $\mathbb{F}^{k, j}:=\left\{\mathscr{F}_{t}^{k, j}: 0 \leq t \leq T\right\}$ be the natural filtration generated by $\left\{A_{t}^{k, j} ; 0 \leq t \leq T\right\}$. One should notice that $\mathbb{F}^{k, j}$ is a discrete-type filtration in the sense that

$$
\mathscr{F}_{t}^{k, j}=\bigvee_{\ell=0}^{\infty}\left(\mathscr{F}_{T_{\ell}^{k, j}}^{k, j} \cap\left\{T_{\ell}^{k, j} \leq t<T_{\ell+1}^{k, j}\right\}\right), \quad 0 \leq t \leq T,
$$

where $\mathscr{F}_{0}^{k, j}=\{\Omega, \emptyset\}$ and $\mathscr{F}_{T_{m}^{k, j}}^{k, j}=\sigma\left(T_{1}^{k, j}, \ldots, T_{m}^{k, j}, \eta_{1}^{k, j}, \ldots\right.$, $\eta_{m}^{k, j}$ ) for $m \geq 1$ and $j=1, \ldots, p$. In (27), $\vee$ denotes the smallest sigma-algebra generated by the union. One can easily check that $\mathscr{F}_{T_{m}^{k, j}}^{k, j}=\sigma\left(A_{s \wedge T_{m}^{k, j}}^{k, j} ; s \geq 0\right)$ and hence

$$
\underset{T_{m}^{k, j}}{\mathscr{F}^{k, j}}=\mathscr{F}_{t}^{k, j} \quad \text { a.s on }\left\{T_{m}^{k, j} \leq t<T_{m+1}^{k, j}\right\} .
$$

With a slight abuse of notation, we write $\mathscr{F}_{t}^{k, j}$ to denote its $\mathbb{Q}$-augmentation satisfying the usual conditions.

Let us now introduce the multidimensional filtration generated by $A^{k}$. Let us consider $\mathbb{F}^{k}:=\left\{\mathscr{F}_{t}^{k} ; 0 \leq t \leq T\right\}$ where $\mathscr{F}_{t}^{k}:=\mathscr{F}_{t}^{k, 1} \otimes \mathscr{F}_{t}^{k, 2} \otimes \cdots \otimes \mathscr{F}_{t}^{k, p}$ for $0 \leq t \leq T$. Let $\mathscr{T}^{k}:=\left\{T_{m}^{k} ; m \geq 0\right\}$ be the order statistics obtained from the family of random variables $\left\{T_{\ell}^{k, j} ; \ell \geq 0 ; j=1, \ldots, p\right\}$. That is, we set $T_{0}^{k}:=0$,

$$
T_{1}^{k}:=\inf _{1 \leq j \leq p}\left\{T_{1}^{k, j}\right\}, \quad T_{n}^{k}:=\inf _{\substack{1 \leq j \leq p \\ m \geq 1}}\left\{T_{m}^{k, j} ; T_{m}^{k, j} \geq T_{n-1}^{k}\right\}
$$

for $n \geq 1$. In this case, $\mathscr{T}^{k}$ is the partition generated by all stopping times defined in (24). The finite-dimensional distribution of $W^{(j)}$ is absolutely continuous for each $j=$ $1, \ldots, p$ and therefore the elements of $\mathscr{T}^{k}$ are almost surely distinct for every $k \geq 1$. The following result is an immediate consequence of our construction. 
Lemma 4. For every $k \geq 1$, the set $\mathscr{T}^{k}$ is a sequence of $\mathbb{F}^{k}$ stopping times such that

$$
\mathscr{F}_{t}^{k}=\mathscr{F}_{T_{n}^{k}}^{k} \quad \text { a.s on }\left\{T_{n}^{k} \leq t<T_{n+1}^{k}\right\},
$$

for each $n \geq 0$ and $k \geq 1$.

Itô representation theorem yields

$$
\mathbb{E}\left[H \mid \mathscr{F}_{t}\right]=\mathbb{E}[H]+\int_{0}^{t} \phi_{u}^{H} d W_{u} ; \quad 0 \leq t \leq T,
$$

where $\phi^{H}$ is a $p$-dimensional $\mathbb{F}$-predictable process such that

$$
\mathbb{E} \int_{0}^{T}\left\|\phi_{t}^{H}\right\|_{\mathbb{R}^{p}}^{2} d t<\infty
$$

The payoff $H$ induces the $\mathbb{Q}$-square-integrable $\mathbb{F}$-martingale $X_{t}:=\mathbb{E}\left[H \mid \mathscr{F}_{t}\right], 0 \leq t \leq T$. We now embed the process $X$ into the filtration $\mathbb{F}^{k}$ by means of the following operator:

$$
\left(\delta^{k} X\right)_{t}:=\sum_{m=0}^{\infty} \mathbb{E}\left[X_{T_{m}^{k}} \mid \mathscr{F}_{T_{m}^{k}}^{k}\right] \mathbb{1}_{\left\{T_{m}^{k} \leq t<T_{m+1}^{k}\right\}} ; \quad 0 \leq t \leq T .
$$

With a slight abuse of notation, we write $\delta^{k} X_{t}$ instead of $\left(\delta^{k} X\right)_{t}$. Since $X$ is an $\mathbb{F}$-martingale, the usual optional stopping theorem and Lemma 4 yield the representation

$$
\delta^{k} X_{t}=\mathbb{E}\left[X_{T} \mid \mathscr{F}_{t}^{k}\right]=\mathbb{E}\left[H \mid \mathscr{F}_{t}^{k}\right], \quad 0 \leq t \leq T .
$$

Therefore, $\delta^{k} X$ is indeed a $\mathbb{Q}$-square-integrable $\mathbb{F}^{k}$ martingale and we will write it as

$$
\begin{aligned}
\delta^{k} X_{t} & =X_{0}+\sum_{m=1}^{\infty} \Delta \delta^{k} X_{T_{m}^{k}} \mathbb{1}_{\left\{T_{m}^{k} \leq t\right\}} \\
& =X_{0}+\sum_{j=1}^{p} \sum_{n=1}^{\infty} \Delta \delta^{k} X_{T_{n}^{k, j}} \mathbb{1}_{\left\{T_{n}^{k, j} \leq t\right\}} \\
& =X_{0}+\sum_{j=1}^{p} \sum_{\ell=1}^{\infty} \frac{\Delta \delta^{k} X_{T_{\ell}^{k, j}}}{\Delta A_{T_{\ell}^{k, j}}^{k, j}} \Delta A_{T_{\ell}^{k, j}}^{k, j} \mathbb{1}_{\left\{T_{\ell}^{k, j} \leq t\right\}} \\
& =X_{0}+\sum_{j=1}^{p} \int_{0}^{t} \mathscr{D}^{j}\left(\delta^{k} X\right)_{u} d A_{u}^{k, j},
\end{aligned}
$$

where

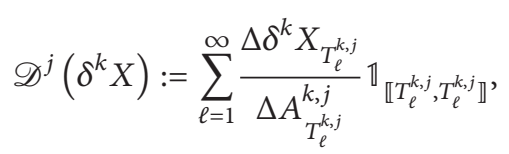

and the integral in (35) is computed in the Lebesgue-Stieltjes sense. For a given $j=1, \ldots, p$ and $\ell \geq 1$, let us define $\tau_{\ell}^{k, j}:=$ $\max \left\{T_{n}^{k} ; T_{n}^{k}<T_{\ell}^{k, j}\right\}$. It is easy to see that

$$
\mathscr{F}_{T_{\ell}^{k, j}}^{k}=\mathscr{F}_{\tau_{\ell}^{k, j}}^{k} \vee \sigma\left(T_{\ell}^{k, j}-T_{\ell-1}^{k, j}, \eta_{\ell}^{k, j}\right),
$$

for $\ell \geq 1$ and $j=1, \ldots, p$. Therefore,

$$
\mathscr{D}^{j}\left(\delta^{k} X\right)_{T_{\ell}^{k, j}}=\frac{\mathbb{E}\left[H \mid \mathscr{F}_{T_{\ell}^{k, j}}^{k}\right]-\mathbb{E}\left[H \mid \mathscr{F}_{\tau_{\ell}^{k, j}}^{k}\right]}{W_{T_{\ell}^{k, j}}^{(j)}-W_{T_{\ell-1}^{k, j}}^{(j)}} ; \quad \ell \geq 1 .
$$

Remark 5. Similar to the univariate case, one can easily check that $\mathbb{F}^{k} \rightarrow \mathbb{F}$ weakly and since $X$ has continuous paths, $\delta^{k} X \rightarrow X$ uniformly in probability as $k \rightarrow \infty$. See Remark 2.1 in [27].

Based on the Dirac process $\mathscr{D}^{j}\left(\delta^{k} X\right)$, we denote

$$
\mathbb{D}^{k, j} X:=\sum_{\ell=1}^{\infty} \mathscr{D}^{j}\left(\delta^{k} X\right)_{T_{\ell}^{k, j}} \mathbb{\square}_{\llbracket T_{\ell}^{k, j}, T_{\ell+1}^{k, j} \mathbb{1}}, \quad k \geq 1, \quad j=1, \ldots, p .
$$

In order to work with nonanticipative hedging strategies, let us now define a suitable $\mathbb{F}^{k}$-predictable version of $\mathbb{D}^{k, j} X$ as follows:

$$
\begin{array}{r}
\mathbf{D}^{k, j} X:=0 \mathbb{1}_{\llbracket 0 \rrbracket}+\sum_{n=1}^{\infty} \mathbb{E}\left[\mathbb{D}^{k, j} X_{T_{n}^{k, j}} \mid \mathscr{F}_{T_{n-1}^{k, j}}^{k}\right] \mathbb{1}_{\rrbracket T_{n-1}^{k, j}, T_{n}^{k, j} \rrbracket} ; \\
k \geq 1, \quad j=1, \ldots, d .
\end{array}
$$

Remark 6. Let $H$ be a contingent claim satisfying (M). Then for a given $j=1, \ldots, p$, we have

$$
\begin{array}{r}
\mathbf{D}^{k, j} X_{t}=\mathbb{E}\left[\frac{\left[\mathbb{E}\left[H \mid \mathscr{F}_{T_{1}^{k, j}}^{k}\right]-\mathbb{E}\left[H \mid \mathscr{F}_{\tau_{1}^{k, j}}^{k}\right]\right.}{W_{T_{1}^{k, j}}^{(j)}-W_{T_{0}^{k, j}}^{(j)}}\right] ; \\
0<t \leq T_{1}^{k, j} .
\end{array}
$$

One should notice that (41) is reminiscent from the usual delta-hedging strategy but the price is shifted on the level of the sigma-algebras jointly with the increments of the driving Brownian motion instead of the pure spot price. For instance, in the one-dimensional case $(p=d=1)$, we have

$$
\mathbf{D}^{k, 1} X_{t}=\mathbb{E}\left[\frac{\mathbb{E}\left[H \mid \mathscr{F}_{T_{1}^{k, 1}}^{k}\right]-\mathbb{E}[H]}{W_{T_{1}^{k, 1}}^{(1)}-W_{T_{0}^{k, 1}}^{(1)}}\right], \quad 0<t \leq T_{1}^{k, 1} .
$$

Identity (41) suggests a natural procedure to approximate pure hedging strategies by means of $\mathbf{D}^{k, 1} X_{T_{1}^{k, 1}} / S_{0} \sigma_{0}$ at time zero. Additional randomness from, for example, stochastic volatilities is encoded by the set of information $\mathscr{F}_{\tau_{1}^{k, j}}^{k}$ which is determined by the Brownian motion hitting times coming from stochastic volatility.

In the next sections, we will construct feasible approximations for the gain process based on ratios (41). We will see that hedging ratios of form (41) will be the key ingredient to recover the gain process in full generality. 
3.2. Weak Approximation for the Hedging Process. Based on (20), (22), and (23), let us denote

$$
\begin{array}{r}
\theta_{t}^{H}:=\phi_{t}^{H, S}\left[\operatorname{diag}\left(S_{t}\right) \sigma_{t}\right]^{-1}, \quad L_{t}^{H}:=\mathbb{E}[H]+\int_{0}^{t} \phi_{\ell}^{H, I} d W_{\ell}^{I} ; \\
0 \leq t \leq T .
\end{array}
$$

In order to shorten notation, we do not write $\left(\phi^{H, \mathbb{Q}, S}, \phi^{H, \mathbb{Q}, I}\right)$ in (43). The main goal of this section is the obtention of bounded variation martingale weak approximations for both gain and cost processes, given, respectively, by

$$
\int_{0}^{t} \theta_{u}^{H} d S_{u}, \quad L_{t}^{H} ; \quad 0 \leq t \leq T .
$$

We assume the trader has some knowledge of the underlying volatility so that the obtention of $\phi^{H, S}$ will be sufficient to recover $\theta^{H}$. The typical example we have in mind is generalized Föllmer-Schweizer decompositions and locally risk minimizing and mean variance strategies as explained in the Introduction. The scheme will be very constructive in such way that all the elements of our approximation will be amenable to a feasible numerical analysis. Under very mild integrability conditions, the weak approximations for the gain process will be translated into the physical measure.

The Weak Topology. In order to obtain approximation results under full generality, it is important to consider a topology which is flexible to deal with nonsmooth hedging strategies $\theta^{H}$ for possibly non-Markovian payoffs $H$ and at the same time justifies Monte Carlo procedures. In the sequel, we make use of the weak topology $\sigma\left(B^{p}, M^{q}\right)$ of the Banach space $B^{P}(\mathbb{F})$ constituted by $\mathbb{F}$-optional processes $Y$ such that

$$
\mathbb{E}\left|Y_{T}^{*}\right|^{p}<\infty
$$

where $Y_{T}^{*}:=\sup _{0 \leq t \leq T}\left|Y_{t}\right|$ and $1 \leq p, q<\infty$ such that $1 / p+$ $1 / q=1$. The subspace of the square-integrable $\mathbb{F}$-martingales will be denoted by $H^{2}(\mathbb{F})$. It will be also useful to work with $\sigma\left(B^{1}, \Lambda^{\infty}\right)$-topology given in [27]. For more details about these topologies, we refer to the works $[27,31,32]$. It turns out that $\sigma\left(B^{2}, M^{2}\right)$ and $\sigma\left(B^{1}, \Lambda^{\infty}\right)$ are very natural notions to deal with generic square-integrable random variables as described in [27].

In the sequel, we recall the following notion of covariation introduced in $[27,30]$.

Definition 7. Let $\left\{Y^{k} ; k \geq 1\right\}$ be a sequence of squareintegrable $\mathbb{F}^{k}$-martingales. One says that $\left\{Y^{k} ; k \geq 1\right\}$ has $\delta$ covariation with respect to $j$ th component of $A^{k}$ if the limit

$$
\lim _{k \rightarrow \infty}\left[Y^{k}, A^{k, j}\right]_{t}
$$

exists weakly in $L^{1}(\mathbb{Q})$ for every $t \in[0, T]$.

The covariation notion in Definition 7 slightly differs from $[27,30]$ because $\left\{Y^{k} ; k \geq 1\right\}$ is not necessarily a sequence of pure jump $\mathbb{F}^{k}$-adapted process. In fact, since we are in the pure martingale case, we will relax such assumption as demonstrated by the following Lemma.

Lemma 8. Let $\left\{Y^{k, j}=\int_{0}^{\cdot} H_{s}^{k, j} d A^{k, j} ; k \geq 1, j=1, \ldots, p\right\}$ be a sequence of stochastic integrals and $Y^{k}:=\sum_{j=1}^{p} Y^{k, j}$. Assume that

$$
\sup _{k \geq 1} \mathbb{E}\left[Y^{k}, Y^{k}\right]_{T}<\infty
$$

Then $Y^{j}:=\lim _{k \rightarrow \infty} Y^{k, j}$ exists weakly in $B^{2}(\mathbb{F})$ for each $j=$ $1, \ldots, p$ with $Y^{j} \in H^{2}(\mathbb{F})$ if and only if $\left\{Y^{k} ; k \geq 1\right\}$ admits $\delta$-covariation with respect to $j$ th component of $A^{k}$. In this case,

$$
\begin{aligned}
& \lim _{k \rightarrow \infty}\left[Y^{k}, A^{k, j}\right]_{t} \\
& \quad=\lim _{k \rightarrow \infty}\left[Y^{k, j}, A^{k, j}\right]_{t} \\
& \quad=\left[Y^{j}, W^{(j)}\right]_{t} \quad \text { weaklyin } L^{1}(\mathbb{Q}) ; t \in[0, T],
\end{aligned}
$$

for $j=1, \ldots, p$.

Proof. Let $Z_{t}^{k}:=\mathbb{E}\left[Y_{t}^{k} \mid \mathscr{F}_{t}\right], 0 \leq t \leq T$, be a sequence of $\mathbb{F}$-square-integrable martingales. Similar to Lemma 4.2 in [30] or Lemma 3.2 in [27], one can easily check that assumption (47) implies that $\left\{Y^{k} ; k \geq 1\right\}$ is $B^{2}(\mathbb{F})$-weakly relatively sequentially compact where all limit points are F-square-integrable martingales. Moreover, since $Y^{k}$ is a square-integrable $\mathbb{F}^{k}$-martingale, we will repeat the same argument given in Lemma 3.5 in [27] to safely state that

$$
\begin{gathered}
\lim _{k \rightarrow \infty}\left[Y^{k_{i}}, A^{k_{i}, j}\right]_{t}=\left[Z, W^{j}\right]_{t} \\
\text { weakly in } L^{1}(\mathbb{Q}) ; \quad 0 \leq t \leq T,
\end{gathered}
$$

for any $B^{2}(\mathbb{F})$-weakly convergent subsequence where $\lim _{i \rightarrow \infty} Y^{k_{i}}=Z \in \mathbf{H}^{2}(\mathbb{F})$. The multidimensional version of the Brownian motion martingale representation theorem allows us to conclude the proof.

In the sequel, we make use of the following notion of weak functional derivative introduced in $[27,30]$.

Definition 9. Let $H$ be a $\mathbb{Q}$-square-integrable contingent claim satisfying $(\mathrm{M})$ and one sets $X_{t}=\mathbb{E}\left[H \mid \mathscr{F}_{t}\right], 0 \leq t \leq T$. We say that $X$ is weakly differentiable if

$$
\mathscr{D}^{j} X:=\lim _{k \rightarrow \infty} \mathbb{D}^{k, j} X \quad \text { exists weakly in } L^{2}(\mathbb{Q} \times \text { Leb })
$$

for each $j=1, \ldots, p$. In this case, we set $\mathscr{D} X:=$ $\left(\mathscr{D}^{1} X, \ldots, \mathscr{D}^{p} X\right)$.

In Leão and Ohashi [27] and Ohashi et al. [30], the authors introduce this notion of differential calculus which proves to be a weak version of the pathwise functional Itô calculus developed by Dupire [28] and further studied by 
Cont and Fournié [29]. We refer the reader to these works for further details. The following result is an immediate consequence of Proposition 3.1 in [30]. See also Th. 4.1 in [27] for the one-dimensional case.

Lemma 10. Let $H$ be a $\mathbb{Q}$-square-integrable contingent claim satisfying $(M)$. Then the $\mathbb{F}$-martingale $X_{t}=\mathbb{E}\left[H \mid \mathscr{F}_{t}\right], 0 \leq$ $t \leq T$, is weakly differentiable and

$$
\mathscr{D} X=\left(\phi^{H, 1}, \ldots, \phi^{H, p}\right) .
$$

In particular,

$$
\begin{array}{r}
\lim _{k \rightarrow \infty} \sum_{j=1}^{d} \int_{0} \mathbb{D}^{k, j} X_{s} d A_{s}^{k, j}=\sum_{j=1}^{d} \int_{0} \phi_{u}^{H, j} d W_{u}^{(j)}=\int_{0} \theta_{u}^{H} d S_{u} \\
\text { weakly in } B^{2}(\mathbb{F}) .
\end{array}
$$

The result in Lemma 10 in not sufficient to implement dynamic hedging strategies based on $\mathbb{D}^{k, j} X, j=1, \ldots, p$. In order to ensure that our hedging strategies are nonanticipative, we need to study the limiting behavior of $\mathbf{D}^{k, j} X$ as $k \rightarrow \infty$. It turns out that they share the same asymptotic behavior as follows. In the sequel, $\int \mathrm{D}^{k, j} X_{s} d A_{s}^{k, j}$ denotes the usual stochastic integral with respect to the square-integrable $\mathbb{F}^{k}$-martingale $A^{k, j}$.

Theorem 11. Let $H$ be a $\mathbb{Q}$-square-integrable contingent claim satisfying $(M)$. Then

$$
\begin{gathered}
\lim _{k \rightarrow \infty} \sum_{j=1}^{d} \int_{0} \mathbf{D}^{k, j} X_{s} d A_{s}^{k, j}=\sum_{j=1}^{d} \int_{0} \phi_{u}^{H, j} d W_{u}^{(j)}=\int_{0} \theta_{u}^{H} d S_{u}, \\
L^{H}=\lim _{k \rightarrow \infty} \sum_{j=d+1}^{p} \int_{0}^{\cdot} \mathbf{D}^{k, j} X_{s} d A_{s}^{k, j}
\end{gathered}
$$

weakly in $B^{2}(\mathbb{F})$. In particular,

$$
\lim _{k \rightarrow \infty} \mathbf{D}^{k, j} X=\phi^{H, j}
$$

weakly in $L^{2}(\mathbb{Q} \times L e b)$ for each $j=1, \ldots, p$.

Proof. We divide the proof into two steps. Throughout this proof $C$ is a generic constant which may defer from line to line.

Step 1. In the sequel, let ${ }^{o, k}(\cdot)$ and ${ }^{p, k}(\cdot)$ be the optional and predictable projections with respect to $\mathbb{F}^{k}$, respectively. See, for example, [31, 33] for further details. Let us consider the $\mathbb{F}^{k}$-martingales given by

$$
M_{t}^{k}:=\sum_{j=1}^{p} M_{t}^{k, j} ; \quad 0 \leq t \leq T,
$$

where

$$
M_{t}^{k, j}:=\int_{0}^{t} \mathbf{D}^{k, j} X_{s} d A_{s}^{k, j} ; \quad 0 \leq t \leq T, j=1, \ldots, p .
$$

We claim that $\sup _{k \geq 1} \mathbb{E}\left[M^{k}, M^{k}\right]_{T}<\infty$. By the very definition,

$$
\begin{aligned}
& \left\{(t, \omega) \in[0, T] \times \Omega ; \Delta\left[A^{k, j}, A^{k, j}\right]_{t}(\omega) \neq 0\right\} \\
& \quad=\bigcup_{n=1}^{\infty} \llbracket T_{n}^{k, j}, T_{n}^{k, j} \rrbracket .
\end{aligned}
$$

Therefore, Jensen inequality yields

$$
\begin{aligned}
& \mathbb{E}\left[M^{k}, M^{k}\right]_{T}= \mathbb{E} \sum_{j=1}^{p} \int_{0}^{T}\left|\mathbf{D}^{k, j} X_{s}\right|^{2} d\left[A^{k, j}, A^{k, j}\right]_{s} \\
& \leq \sum_{j=1}^{p} \mathbb{E} \sum_{n=1}^{\infty} \mathbb{E}\left[\left(\mathbb{D}^{k, j} X_{T_{n}^{k, j}}\right)^{2} \mid \mathscr{F}_{T_{n-1}^{k, j}}^{k}\right] \\
& \times 2^{-2 k} \mathbb{q}_{\left\{T_{n}^{k, j} \leq T\right\}}=: J^{k} .
\end{aligned}
$$

We will write $J^{k}$ in a slightly different manner as follows. In the sequel, for each $t \in(0, T]$, we set $\tau_{t-}^{k, j}:=\max \left\{T_{n}^{k, j} ; T_{n}^{k, j} \leq\right.$ $t\}$ and $\tau_{t+}^{k, j}:=\min \left\{T_{n}^{k, j} ; T_{n}^{k, j}>t\right\}$. Then, we will write

$$
\begin{aligned}
J^{k}= & \mathbb{E}\left\{\sum_{j=1}^{p} \sum_{n=1}^{\infty} \mathbb{E}\left[\left(\mathbb{D}^{k, j} X_{T_{n}^{k, j}}\right)^{2} \mid \mathscr{F}_{T_{n-1}^{k, j}}^{k}\right] 2^{-2 k} \mathbb{1}_{\left\{T_{n-1}^{k, j} \leq T\right\}}\right. \\
& -\sum_{j=1}^{p} \mathbb{E}\left[\left(\mathbb{D}^{k, j} X_{\tau_{T+}^{k, j}}^{k, j} \mid \mathscr{F}_{\tau_{T-}^{k, j}}^{k}\right] 2^{\left.-2 k_{1} \mathbb{1}_{\left\{\tau_{T-}^{k, j} \leq T<\tau_{T+}^{k, j}\right\}}\right\}}\right. \\
= & \sum_{j=1}^{p} \mathbb{E} \sum_{n=1}^{\infty}\left|\Delta \delta^{k} X_{T_{n}^{k, j}}\right|^{2} \mathbb{1}_{\left\{T_{n-1}^{k, j} \leq T\right\}} \\
& -\sum_{j=1}^{p} \mathbb{E}\left[\mathbb{E}\left[\left(\Delta \delta^{k} X_{\tau_{T+}^{k, j}}\right)^{2} \mid \mathscr{F}_{\tau_{T-}^{k, j}}^{k}\right]\right] \mathbb{1}_{\left\{\tau_{T-}^{k, j} \leq T<\tau_{T+}^{k, j}\right\}} .
\end{aligned}
$$

The above identities, Lemma 3.1 in [30], (58), and Remark 5 yield

$$
\limsup _{k \rightarrow \infty} \mathbb{E}\left[M^{k}, M^{k}\right]_{T} \leq \limsup _{k \rightarrow \infty} J^{k}<\infty
$$

Step 2. We claim that for a given $g \in L^{\infty}, t \in[0, T]$, and $j=1 \ldots, p$ we have

$$
\lim _{k \rightarrow \infty} \mathbb{E} g\left[M^{k}-\delta^{k} X, A^{k, j}\right]_{t}=0 .
$$

By using the fact that $\mathbb{D}^{k, j} X$ is $\mathbb{F}^{k}$-optional and $\mathbf{D}^{k, j} X$ is $\mathbb{F}^{k}$ predictable, we will use duality of the $\mathbb{F}^{k}$-optional projection to write

$$
\begin{aligned}
\mathbb{E} g & {\left[M^{k}-\delta^{k} X, A^{k, j}\right]_{t} } \\
= & \mathbb{E} \int_{0}^{t}{ }^{o, k}(g)_{s}\left(\mathbf{D}^{k, j} X_{s}-\mathbb{D}^{k, j} X_{s}\right) d\left[A^{k, j}, A^{k, j}\right]_{s} .
\end{aligned}
$$


In order to prove (61), let us check that

$$
\begin{gathered}
\lim _{k \rightarrow \infty} \mathbb{E} \int_{0}^{t} p, k(g)_{s}\left(\mathbf{D}^{k, j} X_{s}-\mathbb{D}^{k, j} X_{s}\right) d\left[A^{k, j}, A^{k, j}\right]_{s}=0 \\
\lim _{k \rightarrow \infty} \mathbb{E} \int_{0}^{t}\left({ }^{o, k}(g)_{s}-{ }^{p, k}(g)_{s}\right)\left(\mathbf{D}^{k, j} X_{s}-\mathbb{D}^{k, j} X_{s}\right) \\
\times d\left[A^{k, j}, A^{k, j}\right]_{s}=0
\end{gathered}
$$

The same trick we did in (59) together with (57) yields

$$
\begin{aligned}
& \mathbb{E} \int_{0}^{t} p, k(g)_{s}\left(\mathbb{D}^{k, j} X_{s}-\mathbb{D}^{k, j} X_{s}\right) d\left[A^{k, j}, A^{k, j}\right]_{s} \\
& =\mathbb{E}\left[p, k(g)_{\tau_{t+}^{k, j}} \mathbb{D}^{k, j} X_{\tau_{t+}^{k, j}}\right] 2^{-2 k} \mathbb{1}_{\left\{\tau_{t-}^{k, j} \leq t<\tau_{t+}^{k, j}\right\}} \\
& \quad-\mathbb{E}\left[\mathbb{E}\left[p, k(g)_{\tau_{t+}^{k, j}} \mathbb{D}^{k, j} X_{\tau_{t+}^{k, j}} \mid \mathscr{F}_{\tau_{t-}^{k, j}}^{k}\right]\right] 2^{-2 k_{1}} \mathbb{1}_{\left\{\tau_{t-}^{k, j} \leq t<\tau_{t+}^{k, j}\right\}} \longrightarrow 0
\end{aligned}
$$

as $k \rightarrow \infty$ because $X$ has continuous paths (see Remark 5). This proves (63). Now, in order to shorten notation, let us denote the expectation in (64) by $I^{k, j}$. Since $T_{n+1}^{k, j}-T_{n}^{k, j}$ is independent of $\mathscr{F}_{T_{n}^{k, j}}^{k, j}$ with $\mathbb{E}\left|T_{n+1}^{k, j}-T_{n}^{k, j}\right|=2^{-2 k}$, we will write

$$
\begin{aligned}
I^{k, j}= & \mathbb{E} \sum_{n=1}^{\infty}\left({ }^{o, k}(g)_{T_{n}^{k, j}}-{ }^{p, k}(g)_{T_{n}^{k, j}}\right) \\
& \times\left(\mathbb{D}^{k, j} X_{T_{n}^{k, j}}-\mathbb{D}^{k, j} X_{T_{n}^{k, j}}\right)\left(T_{n+1}^{k, j}-T_{n}^{k, j}\right) \mathbb{1}_{\left\{T_{n}^{k, j} \leq t\right\}} \\
= & \mathbb{E} \int_{0}^{t}\left({ }^{o, k, j}(g)_{s}-{ }^{p, k, j}(g)_{s}\right)\left(\widetilde{\mathbf{D}}^{k, j} X_{s}-\mathbb{D}^{k, j} X_{s}\right) d s \\
& +\mathbb{E} \sum_{\ell=0}^{\infty} \int_{t}^{T_{\ell+1}^{k, j}}\left({ }^{o, k, j}(g)_{s}-{ }^{p, k, j}(g)_{s}\right) \\
=: & I_{1}^{k, j}+I_{2}^{k, j},
\end{aligned}
$$

where we set $\widetilde{\mathbf{D}}^{k, j} X_{s}:=\sum_{\ell=1}^{\infty} \mathbf{D}^{k, j} X_{T_{\ell}^{k, j}} \mathbb{1}_{\left\{T_{\ell}^{k, j} \leq s<T_{\ell+1}^{k, j}\right\}}$ and

$$
\begin{array}{ll}
{ }^{o, k, j}(g)_{s}:={ }^{o, k}(g)_{T_{n}^{k, j}} & \text { on }\left\{T_{n}^{k, j} \leq s<T_{n+1}^{k, j}\right\} \\
{ }^{p, k, j}(g)_{s}:={ }^{p, k}(g)_{T_{n}^{k, j}} & \text { on }\left\{T_{n}^{k, j} \leq s<T_{n+1}^{k, j}\right\} ; n \geq 1 .
\end{array}
$$

Again, the independence between $T_{n+1}^{k, j}-T_{n}^{k, j}$ and $\mathscr{F}_{T_{n}^{k, j}}^{k, j}$ together with estimate (60) and $\mathbb{E}\left|T_{n+1}^{k, j}-T_{n}^{k, j}\right|=2^{-2 k}$ yields

$$
\sup _{k \geq 1}\left(\mathbb{E} \int_{0}^{T}\left|\widetilde{\mathbf{D}}^{k, j} X_{s}-\mathbb{D}^{k, j} X_{s}\right|^{2} d s\right)^{1 / 2}<\infty .
$$

Lemma 4.1 in [27], Cauchy-Schwartz inequality, and (68) yield

$$
\begin{aligned}
&\left|I_{1}^{k, j}\right| \leq\left(\mathbb{E} \int_{0}^{T}\left|o, k, j(g)_{s}-{ }^{p, k, j}(g)_{s}\right|^{2} d s\right)^{1 / 2} \\
& \times\left(\mathbb{E} \int_{0}^{T}\left|\widetilde{\mathbf{D}}^{k, j} X_{s}-\mathbb{D}^{k, j} X_{s}\right|^{2} d s\right)^{1 / 2} \\
& \leq C\left(\mathbb{E} \sup _{0 \leq s \leq T}\left|o, k, j(g)_{s}-{ }^{p, k, j}(g)_{s}\right|^{2}\right)^{1 / 2} \longrightarrow 0 \\
& \text { as } k \rightarrow \infty .
\end{aligned}
$$

By the same reasoning, $\left|I_{2}^{k, j}\right| \rightarrow 0$ as $k \rightarrow \infty$, and we conclude that (64) holds. Summing up Steps 1 and 2, we will use Lemmas 8 and 10 to conclude that (53) hold true. It remains to show (54) but this is a straightforward consequence of (48) in Lemma 8 and (61). This concludes the proof of the theorem.

Stronger convergence results can be obtained under path smoothness assumptions for representations $\left(\phi^{H, 1}, \ldots, \phi^{H, p}\right)$. We refer the reader to the Appendix for further details.

\section{Weak Dynamic Hedging}

In this section, we apply Theorem 11 for the formulation of a dynamic hedging strategy starting with a given GKW decomposition

$$
H=\mathbb{E}[H]+\int_{0}^{T} \theta_{t}^{H} d S_{t}+L_{T}^{H},
$$

where $H$ is a $\mathbb{Q}$-square-integrable European-type option satisfying $(\mathrm{M})$ for a given $\mathbb{Q} \in \mathscr{M}^{e}$. The typical examples we have in mind are quadratic hedging strategies with respect to a fully path-dependent option. We recall that when $\mathbb{Q}$ is the minimal martingale measure, then (70) is the generalized Föllmer-Schweizer decomposition so that, under some $\mathbb{P}$ square-integrability conditions on the components of (70), $\theta^{H}$ is the locally risk minimizing hedging strategy (see, e.g., $[4,7])$. In fact, GKW and Föllmer-Schweizer decompositions are essentially equivalent for the market model assumed in Section 2. We recall that decomposition (70) is not sufficient to fully describe mean variance hedging strategies but the additional component rests on the fundamental representation equations as described in the Introduction. See also expression (110) in Section 6.

For simplicity of exposition, we consider a financial market $(\Omega, \mathbf{F}, \mathbb{P})$ driven by a two-dimensional Brownian motion $B$ and a one-dimensional risky asset price process $S$ as described in Section 2. We stress that all results in this section hold for a general multidimensional setting with the obvious modifications.

In the sequel, we denote

$$
\theta^{k, H}:=\sum_{n=1}^{\infty} \frac{\mathbf{D}^{k, 1} X_{T_{n}^{k, 1}}}{\sigma_{T_{n-1}^{k, 1}} S_{T_{n-1}^{k, 1}}} \mathbb{\llbracket}_{n-1}^{k, 1}, T_{n}^{k, 1} \llbracket,
$$

where $\mathbf{D}^{k, 1} X_{T_{n}^{k, 1}}=\mathbb{E}\left[\mathbb{D}^{k, 1} X_{T_{n}^{k, 1}} \mid \mathscr{F}_{T_{n-1}^{k, 1}}^{k}\right]$ for $k, n \geq 1$. 
Corollary 12. For a given $\mathbb{Q} \in \mathscr{M}^{e}$, let $H$ be a $\mathbb{Q}$-squareintegrable claim satisfying $(M)$. Let

$$
H=\mathbb{E}[H]+\int_{0}^{T} \theta_{t}^{H} d S_{t}+L_{T}^{H}
$$

be the correspondent $G K W$ decomposition under $\mathbb{Q}$. If $d \mathbb{P} /$ $d \mathbb{Q} \in L^{1}(\mathbb{P})$ and

$$
\mathbb{E}_{\mathbb{P}} \sup _{0 \leq t \leq T}\left|\int_{0}^{t} \theta_{u}^{H} d S_{u}\right|<\infty
$$

then

$$
\sum_{n=1}^{\infty} \theta_{T_{n-1}^{k, 1}}^{k, H}\left(S_{T_{n}^{k, 1}}-S_{T_{n-1}^{k, 1}}\right) \mathbb{1}_{\left\{T_{n}^{k, 1} \leq \cdot\right\}} \longrightarrow \int_{0}^{\cdot} \theta_{t}^{H} d S_{t} \text { as } k \longrightarrow \infty
$$

in the $\sigma\left(B^{1}, \Lambda^{\infty}\right)$-topology under $\mathbb{P}$.

Proof. We have $\mathbb{E}|d \mathbb{P} / d \mathbb{Q}|^{2}=\mathbb{E}_{\mathbb{P}}|d \mathbb{P} / d \mathbb{Q}|^{2}(d \mathbb{Q} / d \mathbb{P})=\mathbb{E}_{\mathbb{P}}(d \mathbb{P} /$ $d \mathbb{Q})<\infty$. To shorten notation, let $Y_{t}^{k}:=\int_{0}^{t} \mathbf{D}_{s}^{k, 1} X d A_{s}^{k, 1}$ and $Y_{t}:=\int_{0}^{t} \theta_{\ell}^{H} d S_{\ell}$ for $0 \leq t \leq T$. Let $G$ be an arbitrary F-stopping time bounded by $T$ and let $g \in L^{\infty}(\mathbb{P})$ be an essentially $\mathbb{P}$-bounded random variable and $\mathscr{F}_{G}$-measurable. Let $J \in M^{2}$ be a continuous linear functional given by the purely discontinuous $\mathbb{F}$-optional bounded variation process

$$
J_{t}:=g \mathbb{E}\left[\frac{d \mathbb{P}}{d \mathbb{Q}} \mid \mathscr{F}_{G}\right] \mathbb{1}_{\{G \leq t\}} ; \quad 0 \leq t \leq T,
$$

where the duality action $(\cdot, \cdot)$ is given by $(J, N)=\mathbb{E} \int_{0}^{T} N_{s} d J_{s}$, $N \in B^{2}(\mathbb{F})$. See Section 3.1 in [27] for more details. Then Theorem 11 and the fact that $d \mathbb{P} / d \mathbb{Q} \in L^{2}(\mathbb{Q})$ yield

$$
\begin{aligned}
\mathbb{E}_{\mathbb{P}} g Y_{G}^{k} & =\mathbb{E} Y_{G}^{k} g \frac{d \mathbb{P}}{d \mathbb{Q}}=\left(J, Y^{k}\right) \longrightarrow(J, Y) \\
& =\mathbb{E} Y_{G} g \frac{d \mathbb{P}}{d \mathbb{Q}}=\mathbb{E}_{\mathbb{P}} g Y_{G}
\end{aligned}
$$

as $k \rightarrow \infty$. By the very definition,

$$
\begin{aligned}
& \int_{0}^{t} \mathbf{D}^{k, 1} X_{s} d A_{s}^{k, 1} \\
& \quad=\sum_{n=1}^{\infty} \mathbb{E}\left[\mathbb{D}^{k, 1} X_{T_{n}^{k, 1}} \mid \mathscr{F}_{T_{n-1}^{k, 1}}^{k}\right] \Delta A_{T_{n}^{k, 1}}^{k, 1} \mathbb{1}_{\left\{T_{n}^{k, 1} \leq t\right\}} \\
& \quad=\sum_{n=1}^{\infty} \theta_{T_{n-1}^{k, 1}}^{k, H} \sigma_{T_{n-1}^{k, 1}} S_{T_{n-1}^{k, 1}}\left(W_{T_{n}^{k, 1}}^{(1)}-W_{T_{n-1}^{k, 1}}^{(1)}\right) \mathbb{1}_{\left\{T_{n}^{k, 1} \leq t\right\}} \\
& \quad=\sum_{n=1}^{\infty} \theta_{T_{n-1}^{k, H}}^{k, H}\left(S_{T_{n}^{k, 1}}-S_{T_{n-1}^{k, 1}}\right) \mathbb{1}_{\left\{T_{n}^{k, 1} \leq t\right\}} ; \quad 0 \leq t \leq T .
\end{aligned}
$$

Then from the definition of the $\sigma\left(B^{1}, \Lambda^{\infty}\right)$-topology based on the physical measure $\mathbb{P}$, we will conclude the proof.
Remark 13. Corollary 12 provides a nonantecipative Riemman-sum approximation for the gain process $\int_{0}^{\cdot} \theta_{t}^{H} d S_{t}$ in a multidimensional filtration setting where no path regularity of the pure hedging strategy $\theta^{H}$ is imposed. The price we pay is a weak-type convergence instead of uniform convergence in probability. However, from the financial point of view this type of convergence is sufficient for the implementation of Monte Carlo methods in hedging. More importantly, we will see that $\theta^{k, H}$ can be fairly simulated and hence the resulting Monte Carlo hedging strategy can be calibrated from market data.

Remark 14. If one is interested only in convergence at the terminal time $0<T<\infty$, then assumption (73) can be weakened to $\mathbb{E}_{\mathbb{P}}\left|\int_{0}^{T} \theta_{t}^{H} d S_{t}\right|<\infty$. Assumption $\mathbb{E}_{\mathbb{P}}(d \mathbb{P} / d \mathbb{Q})<$ $\infty$ is essential to change the $\mathbb{Q}$-convergence into the physical measure $\mathbb{P}$. One should notice that the associated density process is no longer a $\mathbb{P}$-local-martingale and in general such integrability assumption must be checked case by case. Such assumption holds locally for every underlying Itô risky asset price process. Our numerical results suggest that this property behaves well for a variety of spot price models.

Of course, in practice both the spot prices and the trading dates are not observable at the stopping times so we need to translate our results to a given deterministic set of rebalancing hedging dates.

4.1. Hedging Strategies. In this section, we provide a dynamic hedging strategy based on a refined set of hedging dates $\Pi:=$ $0=s_{0}<\cdots<s_{q-1}<s_{q}=T$ for a fixed integer $q$. For this, we need to introduce some objects. For a given $s_{i} \in \Pi$, we set $W_{s_{i}, t}^{(j)}:=W_{s_{i}+t}^{(j)}-W_{s_{i}}^{(j)}, 0 \leq t \leq T-s_{i}$, for $j=1,2$. Of course, by the strong Markov property of the Brownian motion, we know that $W_{s_{i}}^{(j)}$, is an $\left(\mathscr{F}_{s_{i}, t}^{j}\right)_{0 \leq t \leq T-s_{i}}$-Brownian motion for each $j=1,2$ and is independent of $\mathscr{F}_{s_{i}}^{j}$, where $\mathscr{F}_{s_{i}, t}^{j}:=\mathscr{F}_{s_{i}+t}^{j}$ for $0 \leq t \leq T-s_{i}$. Similar to Section 3.1, we set $T_{s_{i}, 0}^{k, 1}:=0$ and

$$
T_{s_{i}, n}^{k, j}:=\inf \left\{t>T_{s_{i}, n-1}^{k, j} ;\left|W_{s_{i}, t}^{(j)}-W_{s_{i}, T_{s_{i}, n-1}^{k, j}}^{(j)}\right|=2^{-k}\right\} ;
$$$$
n \geq 1, \quad j=1,2 \text {. }
$$

For a given $k \geq 1$ and $j=1,2$, we define $\mathscr{H}_{s_{i}, n}^{k, j}$ as the sigmaalgebra generated by $\left\{T_{s_{i}, \ell}^{k, j} ; 1 \leq \ell \leq n\right\}$ and $W_{s_{i}, T_{s_{i}, \ell}^{k, j}}^{(j)}-W_{s_{i}, T_{s_{i}, \ell-1}^{k, j}}^{(j)}$, $1 \leq \ell \leq n$. We then define the following discrete jumping filtration:

$$
\mathscr{F}_{s_{i}, t}^{k, j}:=\mathscr{H}_{s_{i}, n}^{k, j} \text { a.s on }\left\{T_{s_{i}, n}^{k, j} \leq t<T_{s_{i}, n+1}^{k, j}\right\} .
$$

In order to deal with fully path-dependent options, it is convenient to introduce the following augmented filtration:

$$
\mathscr{G}_{s_{i}, t}^{k, j}:=\mathscr{F}_{s_{i}}^{j} \vee \mathscr{F}_{s_{i}, t}^{k, j} ; \quad 0 \leq t \leq T-s_{i},
$$

for $j=1,2$. The bidimensional information flows are defined by $\mathscr{F}_{s_{i}, t}:=\mathscr{F}_{s_{i}, t}^{1} \otimes \mathscr{F}_{s_{i}, t}^{2}$ and $\mathscr{G}_{s_{i}, t}^{k}:=\mathscr{G}_{s_{i}, t}^{k, 1} \otimes \mathscr{G}_{s_{i}, t}^{k, 2}$ for $0 \leq t \leq$ 
$T-s_{i}$. We set $\mathbb{G}_{s_{i}}^{k}:=\left\{\mathscr{G}_{s_{i}, t}^{k} ; 0 \leq t \leq T-s_{i}\right\}$. We will assume that they satisfy the usual conditions. The piecewise constant martingale projection $A_{s_{i}}^{k, j}$ based on $W_{s_{i}}^{(j)}$ is given by

$$
A_{s_{i}, t}^{k, j}:=\mathbb{E}\left[W_{s_{i}, T-s_{i}}^{(j)} \mid \mathscr{G}_{s_{i}, t}^{k, j}\right] ; \quad 0 \leq t \leq T-s_{i} .
$$

We set $\left\{T_{s_{i}, n}^{k} ; n \geq 0\right\}$ as the order statistic generated by the stopping times $\left\{T_{s_{i}, n}^{k, j} ; j=1,2, n \geq 0\right\}$ similar to (29). define

If $H \in L^{2}(\mathbb{Q})$ and $X_{t}=\mathbb{E}\left[H \mid \mathscr{F}_{t}\right], 0 \leq t \leq T$, then we

$$
\delta_{s_{i}}^{k} X_{t}:=\mathbb{E}\left[H \mid \mathscr{G}_{s_{i}, t}^{k}\right] ; \quad 0 \leq t \leq T-s_{i},
$$

so that the related derivative operators are given by

$$
\mathbb{D}_{s_{i}}^{k, j} X:=\sum_{n=1}^{\infty} \mathscr{D}_{T_{s_{i}, n}^{k, j}}^{j} \delta_{s_{i}}^{k} X \mathbb{\llbracket} T_{s_{i}, n,}^{k, j}, T_{s_{i}, n+1}^{k, j} \llbracket,
$$

where

$$
\mathscr{D}^{j} \delta_{s_{i}}^{k} X:=\sum_{n=1}^{\infty} \frac{\Delta \delta_{s_{i}}^{k} X_{T_{s_{i}, n}^{k, j}}^{k}}{\Delta A_{T_{s_{i}, n}^{k, j}}^{k, j}} \mathbb{1}_{\llbracket T_{s_{i}, n,}^{k, j} T_{s_{i}, n}^{k, j} \rrbracket} ; \quad j=1,2, k \geq 1 .
$$

A $\mathbb{G}_{s_{i}}^{k}$-predictable version of $\mathbb{D}_{s_{i}}^{k, j} X$ is given by

$$
\begin{array}{r}
\mathbf{D}_{s_{i}}^{k, j} X:=0 \mathbb{1}_{\llbracket 0 \rrbracket}+\sum_{n=1}^{\infty} \mathbb{E}\left[\mathbb{D}_{s_{i}}^{k, j} X_{T_{s_{i}, n}^{k, j}} \mid \mathscr{G}_{s_{i}, T_{s_{i}, n-1}^{k, j}}^{k}\right] \mathbb{1}_{\mathbb{\prod} T_{s_{i}, n-1}^{k, j}, T_{s_{i}, n}^{k, j},} ; \\
j=1,2 .
\end{array}
$$

In the sequel, we denote

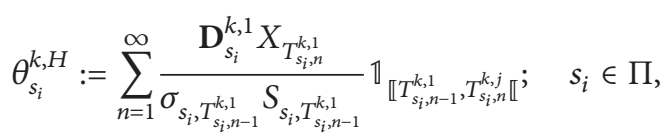

where $\sigma_{s_{i}}$, is the volatility process driven by the shifted filtration $\left\{\mathscr{F}_{s_{i}, t} ; 0 \leq t \leq T-s_{i}\right\}$ and $S_{s_{i}}$, is the risky asset price process driven by the shifted Brownian motion $W_{s_{i}}^{(1)}$.

We are now able to present the main result of this section.

Corollary 15. For a given $\mathbb{Q} \in \mathscr{M}^{e}$, let $H$ be a $\mathbb{Q}$-squareintegrable claim satisfying $(M)$. Let

$$
H=\mathbb{E}[H]+\int_{0}^{T} \theta_{t}^{H} d S_{t}+L_{T}^{H}
$$

be the correspondent $G K W$ decomposition under $\mathbb{Q}$. If $d \mathbb{P} / d \mathbb{Q} \in L^{1}(\mathbb{P})$ and

$$
\mathbb{E}_{\mathbb{P}}\left|\int_{0}^{T} \theta_{u}^{H} d S_{u}\right|<\infty
$$

Then, for any set of trading dates $\Pi=\left\{\left(s_{i}\right)_{i=0}^{q}\right\}$, we have

$$
\begin{aligned}
& \lim _{k \rightarrow \infty} \sum_{s_{i} \in \Pi} \sum_{n=1}^{\infty} \theta_{s_{i}, T_{s_{i}, n-1}^{k, 1}}^{k, H}\left(S_{s_{i}, T_{s_{i}, n}^{k, 1}}-S_{s_{i}, T_{s_{i}, n-1}^{k, 1}}\right) \mathbb{1}_{\left\{T_{s_{i}, n}^{k, 1} \leq s_{i+1}-s_{i}\right\}} \\
& \quad=\int_{0}^{T} \theta_{t}^{H} d S_{t}
\end{aligned}
$$

weakly in $L^{1}$ under $\mathbb{P}$.
Proof. Let $\Pi=\left\{\left(s_{i}\right)_{i=0}^{q}\right\}$ be any set of trading dates where $q$ is a fixed positive integer. To shorten notation, let us define

$$
\begin{aligned}
& R\left(\theta^{k, H}, \Pi, k\right) \\
& \quad:=\sum_{s_{i} \in \Pi} \sum_{n=1}^{\infty} \theta_{s_{i}, T_{s_{i}, n-1}^{k, 1}}^{k, H}\left(S_{s_{i}, T_{s_{i}, n}^{k, 1}}-S_{s_{i}, T_{s_{i}, n-1}^{k, 1}}\right) \mathbb{1}_{\left\{T_{s_{i}, n}^{k, 1} \leq s_{i+1}-s_{i}\right\}}
\end{aligned}
$$

for $k \geq 1$ and $\Pi$. At first, we recall that $\left\{T_{s_{i}, n}^{k, 1}-T_{s_{i}, n-1}^{k, 1} ; n \geq\right.$ $\left.1, s_{i} \in \Pi\right\}$ is an i.i.d sequence with absolutely continuous distribution. In this one-dimensional case, the probability of the set $\left\{T_{s_{i}, n}^{k, 1} \leq s_{i+1}-s_{i}\right\}$ is always strictly positive for every $\Pi$ and $k, n \geq 1$. Hence, $R\left(\theta^{k, H}, \Pi, k\right)$ is a nondegenerate subset of random variables. By making a change of variable on the Itô integral, we will write

$$
\begin{aligned}
\int_{0}^{T} \theta_{t}^{H} d S_{t} & =\int_{0}^{T} \phi_{t}^{H, 1} d W_{t}^{(1)}=\sum_{s_{i} \in \Pi} \int_{s_{i}}^{s_{i+1}} \phi_{t}^{H, 1} d W_{t}^{(1)} \\
& =\sum_{s_{i} \in \Pi} \int_{0}^{s_{i+1}-s_{i}} \phi_{s_{i}+t}^{H, 1} d W_{s_{i}, t}^{(1)} .
\end{aligned}
$$

Let us fix $\mathbb{Q} \in \mathscr{M}^{e}$. By the very definition,

$$
R\left(\theta^{k, H}, \Pi, k\right)=\sum_{s_{i} \in \Pi} \int_{0}^{s_{i+1}-s_{i}} \mathbf{D}_{s_{i}}^{k, 1} X_{\ell} d A_{s_{i}, \ell}^{k, 1} \quad \text { under } \mathbb{Q} \text {. }
$$

Now we notice that Theorem 11 holds for the twodimensional Brownian motion $\left(W_{s_{i}}^{(1)}, W_{s_{i}}^{(2)}\right)$, for each $s_{i} \in \Pi$ with the discretization of the Brownian motion given by $A_{s_{i}}^{k, 1}$. Moreover, using the fact that $\mathbb{E}|d \mathbb{P} / d \mathbb{Q}|^{2}<\infty$ and repeating the argument given by (77) restricted to the interval $\left[s_{i}, s_{i+1}\right)$, we have

$$
\begin{aligned}
\lim _{k \rightarrow \infty} R\left(\theta^{k, H}, \Pi, k\right) & =\sum_{s_{i} \in \Pi} \lim _{k \rightarrow \infty} \int_{0}^{s_{i+1}-s_{i}} \mathbf{D}_{s_{i}}^{k, 1} X_{\ell} d A_{s_{i}, \ell}^{k, 1} \\
& =\int_{0}^{T} \theta_{t}^{H} d S_{t},
\end{aligned}
$$

weakly in $L^{1}(\mathbb{P})$ for each $\Pi$. This concludes the proof.

Remark 16. In practice, one may approximate the gain process by a nonantecipative strategy as follows. Let $\Pi$ be a given set of trading dates on the interval $[0, T]$ so that $|\Pi|=$ $\max _{0 \leq i \leq q}\left|s_{i}-s_{i-1}\right|$ is small. We take a large $k$ and we perform a nonantecipative buy-and-hold-type strategy among the trading dates $\left[s_{i}, s_{i+1}\right) ; s_{i} \in \Pi$, in the full approximation (90) which results in

$$
\begin{array}{r}
\sum_{s_{i} \in \Pi} \theta_{s_{i}, 0}^{k, H}\left(S_{s_{i}, s_{i+1}-s_{i}}-S_{s_{i}, 0}\right) \quad \text { where } \\
\theta_{s_{i}, 0}^{k, H}=\frac{\mathbb{E}\left[\mathbb{D}_{s_{i}}^{k, 1} X_{T_{s_{1}, 1}^{k, 1}} \mid \mathscr{F}_{s_{i}}\right]}{\sigma_{s_{i}, 0} S_{s_{i}, 0}} ; \quad s_{i} \in \Pi .
\end{array}
$$

Convergence (89) implies that approximation (94) results in unavoidable hedging errors with respect to the gain process 
due to the discretization of the dynamic hedging, but we do not expect large hedging errors provided that $k$ is large and $|\Pi|$ is small. Hedging errors arising from discrete hedging in complete markets are widely studied in the literature. We do not know optimal rebalancing dates in this incomplete market setting, but simulation results presented in Section 6 suggest that homogeneous hedging dates work very well for a variety of models with and without stochastic volatility. A more detailed study is needed in order to get more precise relations between $\Pi$ and the stopping times, a topic which will be further explored in a future work.

Let us now briefly explain how the results of this section can be applied to well-known quadratic hedging methodologies.

Generalized Föllmer-Schweizer. If one takes the minimal martingale measure $\widehat{\mathbb{P}}$, then $L^{H}$ in $(70)$ is a $\mathbb{P}$-local martingale and is orthogonal to the martingale component of $S$. Due this orthogonality and the zero mean behavior of the cost $L^{H}$, it is still reasonable to work with generalized FöllmerSchweizer decompositions under $\mathbb{P}$ without knowing a priori the existence of locally risk minimizing hedging strategies.

Local Risk Minimization. One should notice that if $\int \theta^{H} d S \in$ $B^{2}(\mathbb{F}), L^{H} \in B^{2}(\mathbb{F})$ under $\mathbb{P}$ and $d \widehat{\mathbb{P}} / d \mathbb{P} \in L^{2}(\mathbb{P})$, then $\theta^{H}$ is the locally risk minimizing trading strategy and (70) is the Föllmer-Schweizer decomposition under $\mathbb{P}$.

Mean Variance Hedging. If one takes $\widetilde{\mathbb{P}}$, then the mean variance hedging strategy is not completely determined by the GKW decomposition under $\widetilde{\mathbb{P}}$. Nevertheless, Corollary 15 still can be used to approximate the optimal hedging strategy by computing the density process $\widetilde{Z}$ based on the so-called fundamental equations derived by Hobson [16]. See (5) and (6) for details. For instance, in the classical Heston model, Hobson derives analytical formulas for $\widetilde{\zeta}$. See (110) in Section 6.

Hedging of Fully Path-Dependent Options. The most interesting application of our results is the hedging of fully pathdependent options under stochastic volatility. For instance, if $H=\Phi\left(\left\{S_{t} ; 0 \leq t \leq T\right\}\right)$, then Corollary 15 and Remark 16 jointly with the above hedging methodologies allow us to dynamically hedge the payoff $H$ based on (94). The conditioning on the information flow $\left\{\mathscr{F}_{s_{i}} ; s_{i} \in \Pi\right\}$ in the hedging strategy $\theta_{\text {hedg }}^{k, H}:=\left\{\theta_{s_{i}}^{k, H} ; s_{i} \in \Pi\right\}$ encodes the continuous monitoring of a path-dependent option. For each hedging date $s_{i}$, one has to incorporate the whole history of the price and volatility until such date in order to get an accurate description of the hedging. If $H$ is not pathdependent, then the information encoded by $\left\{\mathscr{F}_{s_{i}} ; s_{i} \in \Pi\right\}$ in $\theta_{\text {hedg }}^{k, H}$ is only crucial at time $s_{i}$.

Next, we provide the details of the Monte Carlo algorithm for the approximating pure hedging strategy $\theta_{\text {hedg }}^{k, H}=$ $\left\{\theta_{s_{i}, 0}^{k, H} ; s_{i} \in \Pi\right\}$.

\section{The Algorithm}

In this section we present the basic algorithm to evaluate the hedging strategy for a given European-type contingent claim $H \in L^{2}(\mathbb{Q})$ satisfying assumption (M) for a fixed $\mathbb{Q} \in \mathscr{M}^{e}$ at a terminal time $0<T<\infty$. The core of the algorithm is the simulation of the stochastic derivative $\mathbf{D}^{k, j} X$

$$
\mathbb{E}\left[\frac{\mathbb{E}\left[H \mid \mathscr{F}_{T_{1}^{k, j}}^{k}\right]-\mathbb{E}\left[H \mid \mathscr{F}_{\tau_{1}^{k, j}}^{k}\right]}{A_{T_{1}^{k, j}}^{k, j}}\right], \quad j=1, \ldots, d,
$$

for $d \leq p$. Recall that $\mathbb{F}^{k}$ is a discrete jumping filtration generated by the i.i.d families of Bernoulli and absolutely continuous random variables given, respectively, by $\left\{\eta_{n}^{k, j} ; n \geq\right.$ $1,1 \leq j \leq p\}$ and $\left\{T_{n}^{k, j}-T_{n-1}^{k, j} ; n \geq 1,1 \leq j \leq p\right\}$ which are amenable to an exact simulation by using Burq and Jones [8]. By considering the payoff $H$ as a functional of $A^{k, 1}, \ldots, A^{k, p}$, this section explains how to perform a concrete and feasible Monte Carlo method to obtain the hedging strategies $\theta^{H}$.

In the sequel, we fix the discretization level $k \geq 1$.

Step 1 (simulation of the stopping times $\left\{T_{\ell}^{k, j} ; j=1, \ldots, p\right.$; $\ell \geq 1\}$ and the step processes $\left.\left\{A^{k, j} ; j=1, \ldots, p\right\}\right)$

(1) One generates the increments $\left\{T_{\ell}^{k, j}-T_{\ell-1}^{k, j} ; \ell \geq 1\right\}$ according to the algorithm described by Burq and Jones [8] and, consequently, the $\mathbb{F}^{k, j}$-stopping times $\left\{T_{\ell}^{k, j} ; \ell \geq 1\right\}$ for every $j=1, \ldots, p$, such that all the $\mathbb{F}^{k, j}$-stopping times $T_{\ell}^{k, j} \leq T$.

(2) One simulates the i.i.d family $\eta^{k, j}=\left\{\eta_{\ell}^{k, j} ; \ell \geq 1\right\}$ independently of $\left\{T_{\ell}^{k, j} ; \ell \geq 1\right\}$, according to the Bernoulli random variable $\eta_{1}^{k, j}$ with parameter $1 / 2$ for $i=-1,1$. This simulates the step process $A^{k, j}$ for $j=1, \ldots, p$.

In the next step we need to simulate $H$ based on approximations of the discounted price process $\left\{S_{t}^{i} ; 0 \leq t \leq\right.$ $T ; i=1, \ldots, d\}$ as follows.

Step 2 (simulation of the discounted stock price process $\left.\left\{S^{i} ; i=1, \ldots, d\right\}\right)$. Suppose that, using Step 1, we have the partitions $\mathscr{T}^{k, j}$, the family $\eta^{k, j}$, and the step processes $A^{k, j}$ for $j=1, \ldots, d, d+1, \ldots, p$. The following steps show how to compute approximations to the discounted stock price prices $S^{i}, i=1, \ldots, d$, and the payoff function $H$.

(1) We consider the order statistics $\mathscr{T}^{k}$ generated by all stopping times as defined by (29). This is the finest partition generated by all partitions $\mathscr{T}^{k, j}$.

(2) We apply some appropriate method to evaluate an approximation $S^{k, i}$ of the discounted price $S^{i}$ for $i=$ $1, \ldots, d$, where $S^{k, i}$ is a functional of the noisy $A^{k}=$ $\left(A^{k, 1}, \ldots, A^{k, p}\right)$. Generally speaking, we work with some Itô-Taylor expansion method driven by $A^{k}$. 
(3) Based on the approximation for $S^{k}$, we calculate the approximation for the payoff $\widehat{H}$ as follows: $\widehat{H}^{k}=$ $\Phi\left(S^{k, j} ; 1 \leq j \leq p\right)$.

Next, we describe the crucial step in the algorithm: the simulation of the stochastic derivative described by (41).

Step 3 (simulation of the stochastic derivative $\mathbf{D}^{k, j} X_{T_{1}^{k, j}}$ ). We recall that

$$
\mathbf{D}^{k, j} X_{T_{1}^{k, j}}=\mathbb{E}\left[\frac{\mathbb{E}\left[H \mid \mathscr{F}_{T_{1}^{k, j}}^{k}\right]-\mathbb{E}\left[H \mid \mathscr{F}_{\tau_{1}^{k, j}}^{k}\right]}{A_{T_{1}^{k, j}}^{k, j}}\right],
$$

where $\tau_{1}^{k, j}=\max \left\{T_{n}^{k} ; T_{n}^{k}<T_{1}^{k, j}\right\}$, and $\mathscr{F}_{T_{1}^{k, j}}^{k}$ is given by

$$
\mathscr{F}_{T_{1}^{k, j}}^{k}=\mathscr{F}_{\tau_{1}^{k, j}}^{k} \vee \sigma\left(T_{1}^{k, j}, \eta_{1}^{k, j}\right) .
$$

In the sequel, $t_{\ell}^{k, j}$ denotes the realization of $T_{\ell}^{k, j}$ by means of Step 1 and $t_{\ell}^{k}$ denotes the realization of $T_{\ell}^{k}$ based on the finest random partition $\mathscr{T}^{k}$. Moreover, any sequence $\left(t_{1}^{k}<\right.$ $\left.t_{2}^{k}<\cdots<t_{1}^{k, j}\right)$ encodes the information generated by the realization of $\mathscr{T}^{k}$ until the first hitting time of the $j$ th partition. In addition, we denote $t_{1-}^{k, j}$ as the last time in the finest partition before $t_{1}^{k, j}$. For each $(k, n) \in \mathbb{N} \times \mathbb{N}$, let $\left(v_{1, k}^{n}, v_{2, k}^{n}\right) \in\{1, \ldots, p\} \times \mathbb{N}$ be the unique random pair which realizes

$$
T_{n}^{k}=T_{v_{2, k}^{n}}^{k, v_{1, k}^{n}} \quad \text { a.s, } k, n \geq 1
$$

Based on these quantities, we define $\bar{\eta}_{t_{n}^{k}}^{k}$ as the realization of the random variable $\eta_{v_{2, k}^{n}}^{k, v_{1, k}^{n}}$, where $\left\{\eta_{m}^{k, j} ; 1 \leq j \leq p, m \geq 1\right\}$ is given by (26).

In the sequel, $\widehat{\mathbb{E}}$ denotes the conditional expectation computed in terms of the Monte Carlo method.

(1) For every $j=1, \ldots, d$ we compute

$$
\begin{aligned}
& \widehat{\mathbb{D}}^{k, j} X_{T_{1}^{k, j}}:= \frac{1}{2^{-k} k_{1}^{k, j}}\left\{\widehat{\mathbb{E}}\left[H \mid \mathscr{F}_{T_{1}^{k, j}}^{k}\right]-\widehat{\mathbb{E}}\left[H \mid \mathscr{F}_{\tau_{1}^{k, j}}^{k}\right]\right\} \\
&=\frac{1}{2^{-k} \bar{\eta}_{1}^{k, j}}\left\{\widehat{\mathbb{E}}\left[H \mid\left(t_{1}^{k}, \bar{\eta}_{t_{1}^{k}}^{k}\right), \ldots,\left(t_{1}^{k, j}, \bar{\eta}_{t_{1}^{k, j}}^{k}\right)\right]\right. \\
&\left.\quad-\widehat{\mathbb{E}}\left[H \mid\left(t_{1}^{k}, \bar{\eta}_{t_{1}^{k}}^{k}\right), \ldots,\left(t_{1-}^{k, j}, \bar{\eta}_{t_{1-}^{k, j}}^{k}\right)\right]\right\},
\end{aligned}
$$

where $\bar{\eta}_{1}^{k, j}$ in (99) denotes the realization of the Bernoulli variable $\eta_{1}^{k, j}$.

(2) We define the stochastic derivative

$$
\widehat{\phi}_{0}^{H, S}:=\left(\widehat{\mathbb{D}}^{k, 1} X_{T_{1}^{k, 1}}, \ldots, \widehat{\mathbb{D}}^{k, d} X_{T_{1}^{k, d}}\right) .
$$

(3) We compute $\bar{\theta}_{0}^{H}$ as

$$
\bar{\theta}_{0}^{H}:=\left(\widehat{\phi}_{0}^{H, S}\right)^{\top}\left[\operatorname{diag}\left(S_{0}\right) \sigma_{0}\right]^{-1} .
$$

(4) Repeat these steps several times and calculate the pure hedging strategy as the mean of all $\bar{\theta}_{0}^{H}$. Consider

$$
\widehat{\theta}_{0}^{H}:=\text { mean of } \bar{\theta}_{0}^{H} \text {. }
$$

Quantity (102) is a Monte Carlo estimate of $\theta_{0}^{H}$.

Remark 17. The Monte Carlo simulation of (99) is performed by considering the payoff $H=\Phi\left(S^{j} ; 1 \leq j \leq p\right)$ as a functional $\Phi\left(S^{k, j} ; 1 \leq j \leq p\right)$ of the noisy $A^{k}=$ $\left(A^{k, 1}, \ldots, A^{k, p}\right)$ in terms of any Itô-Taylor/Euler-Maruyama scheme.

Remark 18. In order to compute the hedging strategy $\theta^{H}$ over a trading period $\left\{s_{i} ; i=0, \ldots, q\right\}$, one performs Algorithms 1 , 2 and 3 (see Appendix) but based on the shifted filtration and the Brownian motions $W_{s_{i}}^{(j)}$ for $j=1, \ldots, p$ as described in Section 4.1.

Remark 19. In practice, one has to calibrate the parameters of a given stochastic volatility model based on liquid instruments such as vanilla options and volatility surfaces. With those parameters at hand, the trader must follow steps (99) and (102). The hedging strategy is then given by calibration and the computation of quantity (102) over a trading period.

\section{Numerical Analysis and Discussion of the Methods}

In this section, we provide a detailed analysis of the numerical scheme proposed in this work.

6.1. Multidimensional Black-Scholes Model. At first, we consider the classical multidimensional Black-Scholes model with as many risky stocks as underlying independent random factors to be hedged $(d=p)$. In this case, there is only one equivalent local martingale measure, the hedging strategy $\theta^{H}$ is given by (43), and the cost is just the option price. To illustrate our method, we study a very special type of exotic option: a BLAC (Basket Lock Active Coupon) down-and-out barrier option whose payoff is given by

$$
H=\prod_{i \neq j} \mathbb{1}_{\left\{\min _{s \in[0, T]} S_{s}^{i} \vee \min _{s \in[0, T]} S_{s}^{j}>L\right\}} .
$$

It is well known that, for this type of option, there exists a closed formula for the hedging strategy. Moreover, it satisfies the assumptions of Theorem A.2. See, for example, Bernis et al. [34] for some formulas.

For comparison purposes with Bernis et al. [34], we consider $d=5$ underlying assets, $r=0 \%$ for the interest rate, and $T=1$ year for the maturity time. For each asset, we set initial values $S_{0}^{i}=100,1 \leq i \leq 5$, and we compute the hedging 


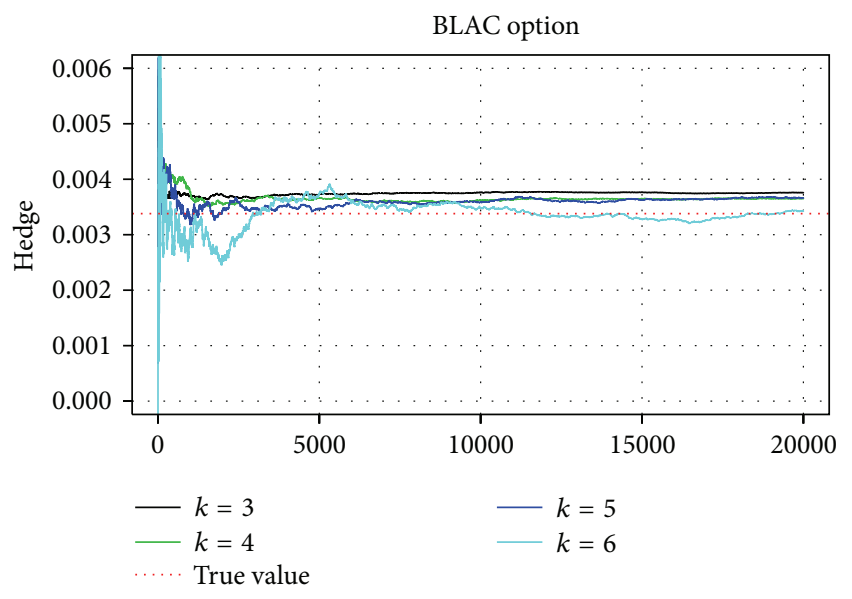

Figure 1: Monte Carlo hedging strategy of a BLAC down-and-out option for a 5-dimensional Black-Scholes model.

strategy with respect to the first asset $S^{1}$ with discretization level $k=3,4,5,6$ and 20000 simulations.

Following the work [34], we consider the volatilities of the assets given by $\left\|\sigma^{1}\right\|=35 \%,\left\|\sigma^{2}\right\|=35 \%,\left\|\sigma^{3}\right\|=38 \%,\left\|\sigma^{4}\right\|=$ $35 \%$, and $\left\|\sigma^{5}\right\|=40 \%$ and the correlation matrix defined by $\rho_{i j}=0,4$ for $i \neq j$, where $\sigma^{i}=\left(\sigma_{i 1}, \ldots, \sigma_{i 5}\right)^{\top}$, and we use the barrier level $L=76$. In Table 1, we present the numerical results based on the Algorithms 1, 2 and 3 for the pointwise hedging strategy $\theta^{H}$ at time $t=0$.

Table 1 reports the difference between the true and the estimated hedging value, the standard error = standard deviation/(number of simulations) $)^{1 / 2}$, the $\%$ error $=$ difference/true valor, and the lower (LL) and upper limits (UL) of the $95 \%$ confidence interval for the empirical mean of the estimated pointwise hedging strategies at time $t=0$. Due to Theorem A.2, we expect that when the discretization level $k$ increases, we obtain results closer to the true value and this is what we find in Monte Carlo experiments, confirmed by the small \% error when using $k=6$. We also emphasize that when $k=6$, the confidence interval contains the true value 0.00338 , and we can really assume the convergence of the algorithm.

In Figure 1, we plot the average hedging estimates with respect to the number of simulations. One should notice that when $k$ increases, the standard error also increases, which suggests more simulations for higher values of $k$.

6.2. Average Hedging Errors. Next, we present some average hedging error results for two well-known nonconstant volatility models: the constant elasticity of variance (CEV) model and the classical Heston stochastic volatility model [18]. The typical examples we have in mind are the generalized Föllmer-Schweizer, local risk minimization, and mean variance hedging strategies, where the optimal hedging strategies are computed by means of the minimal martingale measure and the variance optimal martingale measure, respectively.
We analyze the one-touch one-dimensional European-type contingent claims as follows:

$$
\text { One-touch option: } H=\mathbb{1}_{\left\{\max _{t \in[0, T]} S_{t}>105\right\}} \text {. }
$$

By using the Algorithms 1, 2 and 3, we compute the error committed by approximating the payoff $H$ by $\widehat{\mathbb{E}}_{\mathbb{Q}}[H]+$ $\sum_{i=0}^{n-1} \widehat{\theta}_{t_{i}, 0}^{k, H}\left(S_{t_{i}, t_{i+1}-t_{i}}-S_{t_{i}, 0}\right)$. This error will be called hedging error. The computation of this error is summarized in the following steps.

\section{Computation of the Average Hedging Error}

(1) We first simulate paths under the physical measure and compute the payoff $H$.

(2) Then, we consider some deterministic partition of the interval $[0, T]$ into $n$ (number of hedging strategies in the period) points $t_{0}, t_{1}, \ldots, t_{n-1}$ such that $t_{i+1}-t_{i}=$ $T / n$, for $i=0, \ldots, n-1$.

(3) One simulates, at time $t_{0}=0$, the option price $\widehat{\mathbb{E}}_{\mathbb{Q}}[H]$ and the initial hedging estimate $\hat{\theta}_{0,0}^{k, H}$ through (100), (101), and (102) under a fixed $\mathbb{Q} \in \mathscr{M}^{e}$. We follow the Algorithms 1, 2 and 3.

(4) We simulate $\hat{\theta}_{t_{i}, 0}^{k, H}$ by means of the shifting argument based on the strong Markov property of the Brownian motion as described in Section 4.1.

(5) We compute $\widehat{H}$ by

$$
\widehat{H}:=\widehat{\mathbb{E}}_{\mathbb{Q}}[H]+\sum_{i=0}^{n-1} \widehat{\theta}_{t_{i}, 0}^{k, H}\left(S_{t_{i}, t_{i+1}-t_{i}}-S_{t_{i}, 0}\right) .
$$

(6) We compute the hedging error estimate $\hat{\gamma}$ given by $\widehat{\gamma}:=|H-\widehat{H}|$.

(7) We compute the average hedging error given by AV := $(1 / M) \sum_{\ell=1}^{M} \hat{\gamma}_{\ell}$ where $\widehat{\gamma}_{\ell}$ is the hedging error at the $\ell$ th scenario and $M$ is the total number of scenarios used in the experiment.

(8) We compute $E(\mathrm{AV}):=100 \times \mathrm{AV} / \widehat{\mathbb{E}}_{\mathbb{Q}}[H]$.

Remark 20. When no locally risk minimizing strategy is available, we also expect to obtain low average hedging errors when dealing with generalized Föllmer-Schweizer decompositions due to the orthogonal martingale decomposition. In the mean variance hedging case, two terms appear in the optimal hedging strategy: the pure hedging component $\theta^{H, \widetilde{P}}$ of the GKW decomposition under the optimal variance martingale measures $\widetilde{\mathbb{P}}$ and $\widetilde{\zeta}$ as described by (5) and (6). For the Heston model, $\widetilde{\zeta}$ was explicitly calculated by Hobson [16]. We have used his formula in our numerical simulations jointly with $\widehat{\theta}^{k, H}$ under $\widetilde{\mathbb{P}}$ in the calculation of the mean variance hedging errors. See expression (110) for details.

6.2.1. Constant Elasticity of Variance (CEV) Model. The discounted risky asset price process described by the CEV model under the physical measure is given by

$$
d S_{t}=S_{t}\left[\left(b_{t}-r_{t}\right) d t+\sigma S_{t}^{(\beta-2) / 2} d B_{t}\right], \quad S_{0}=s,
$$


TABLE 1: Monte Carlo hedging strategy of a BLAC down-and-out option for a 5-dimensional Black-Scholes model.

\begin{tabular}{cccccccc}
\hline$k$ & Hedging & St. error & LL & UL & True value & Difference & $\%$ error \\
\hline 3 & 0.00376 & $2.37 \times 10^{-5}$ & 0.00371 & 0.00380 & 0.00338 & 0.00038 \\
4 & 0.00365 & $4.80 \times 10^{-5}$ & 0.00356 & 0.00374 & 0.00338 & 0.00027 & $8.03 \%$ \\
5 & 0.00366 & $9.31 \times 10^{-5}$ & 0.00348 & 0.00384 & 0.00338 & 0.00028 \\
6 & 0.00342 & $1.82 \times 10^{-4}$ & 0.00306 & 0.00378 & 0.00338 & 0.00004 & $8.35 \%$ \\
\hline
\end{tabular}

where $B$ is a $\mathbb{P}$-Brownian motion. The instantaneous Sharpe ratio is $\psi_{t}=\left(b_{t}-r_{t}\right) /\left(\sigma S_{t}^{(\beta-2) / 2}\right)$ such that the model can be rewritten as

$$
d S_{t}=\sigma_{t} S_{t}^{\beta / 2} d W_{t}
$$

where $W$ is a $\mathbb{Q}$-Brownian motion and $\mathbb{Q}$ is the equivalent local martingale measure. In this Monte Carlo experiment, we consider a total number of scenarios $M$ equal to 1000 with the following parameters: the barrier for the one-touch option in (104) is 105, $r=0$ for the interest rate, $b=0.01$, $T=1$ (month) for the maturity time, $\sigma=0.2, S_{0}=$ 100 , and $\beta=1.6$ such that the constant of elasticity is -0.4 . We simulate the average hedging errors by considering discretization levels $k=3,4,5$. We perform $11,16,22$, and 44 hedging strategies along the interval $[0, T]$. We observe that, supposing 22 business days per month, we can assume that 11,22 , and 44 hedging strategies on the interval $[0,1]$ correspond to one hedging strategy for every two days, one hedging strategy per day, and two hedging strategies per day, respectively. From Corollary 15, we know that this procedure is consistent.

Table 2 reports the average hedging errors for the onetouch option. It provides the standard error $=$ standard deviation of $\left\{\widehat{\gamma}_{i} ; 1 \leq i \leq M\right\} /(\text { total number of scenarios } M)^{1 / 2}$, the $\%$ error $=E(\mathrm{AV})$, the lower (LL) and upper limits (UL) of the $95 \%$ confidence interval for $\mathrm{AV}$, and the price of the option. It is important to notice that when $k$ increases, the percentage error $E(\mathrm{AV})$ decreases, which is expected due to the weak convergence results of this paper. We also point out that for $k=5$ all the $95 \%$ confidence intervals contain the zero. Moreover, we notice that as the number of hedging strategies increases, the standard error becomes smaller.

6.2.2. Heston's Stochastic Volatility Model. Here we consider two types of hedging methodologies: local risk minimization and mean variance hedging strategies as described in the Introduction and Remark 20. The Heston dynamics of the discounted price under the physical measure is given by

$$
\begin{aligned}
& d S_{t}=S_{t}\left(b_{t}-r_{t}\right) \Sigma_{t} d t+S_{t} \sqrt{\Sigma_{t}} d B_{t}^{(1)}, \\
& d \Sigma_{t}=2 \kappa\left(\theta-\Sigma_{t}\right) d t+2 \sigma \sqrt{\Sigma_{t}} d Z_{t}, \quad 0 \leq t \leq T,
\end{aligned}
$$

where $Z=\rho B^{(1)}+\bar{\rho} B_{t}^{(2)}, \bar{\rho}=\sqrt{1-\rho^{2}},\left(B^{(1)}, B^{(2)}\right)$ is a pair of two independent $\mathbb{P}$-Brownian motions, and $\kappa, m, \beta_{0}, \mu$ are suitable constants in order to have a well-defined Markov process (see, e.g., $[16,18])$. Alternatively, we can rewrite the dynamics as

$$
\begin{aligned}
& d S_{t}=S_{t} Y_{t}^{2}\left(b_{t}-r_{t}\right) d t+S_{t} Y_{t} d B_{t}^{(1)}, \\
& d Y_{t}=\kappa\left(\frac{m}{Y_{t}}-Y_{t}\right) d t+\sigma d Z_{t}, \quad 0 \leq t \leq T,
\end{aligned}
$$

where $Y=\sqrt{\Sigma_{t}}$ and $m=\theta-\left(\sigma^{2} / 2 \kappa\right)$.

Local Risk Minimization. For comparison purposes with Heath et al. [4], we consider the hedging of a European put option $H$ written on a Heston model with correlation parameter $\rho=0$. We set $S_{0}=100$, strike price $K=100$, and $T=1$ (month) and we use discretization levels $k=3,4$, and 5 . We set the parameters $\kappa=2.5, \theta=0.04, \rho=0, \sigma=0.3, r=0$, and $Y_{0}=0.02$. The hedging strategy $\theta^{H, \widehat{\mathbb{P}}}$ based on the local risk minimization methodology is bounded with continuous paths so that Theorem A.2 applies to this case. Moreover, as described by Heath et al. [4], $\theta^{H, \widehat{\mathbb{P}}}$ can be obtained by a PDE numerical analysis.

Table 3 presents the results of the hedging strategy $\widehat{\theta}_{0,0}^{k, H}$ by using Algorithms 1, 2 and 3. Figure 2 provides the Monte Carlo hedging strategy with respect to the number of simulations of order 10000 . We notice that our results agree with the results obtained by Heath et al. [4] by PDE methods. In this case, the true value of the hedging at time $t=0$ is approximately -0.44 . Table 3 provides the standard errors related to the computed hedging strategy and the Monte Carlo prices.

Hedging with Generalized Föllmer-Schweizer Decomposition for One-Touch Option. Based on Corollary 15, we also present the averaging hedging error associated with one-touch options written on a Heston model with nonzero correlation. We consider a total number of scenarios $M=1000$ and we set $\kappa=3.63, \theta=0.04, \rho=-0.53, \sigma=0.3, r=0$, $b=0.01, Y_{0}=0.3$, and $S_{0}=100$ where the barrier is 105 . We simulate the average hedging error along the interval $[0,1]$ with discretization levels $k=3,4$. We compute 22 and 44 hedging strategies in the period (which corresponds to one and two hedging strategies per day, resp.). The average hedging error results are summarized in Table 4 . It provides the standard error (St. error) $=$ standard deviation of $\left\{\widehat{\gamma}_{\ell} ; 1 \leq\right.$ $\ell \leq M\} /(\text { total number of scenarios } M=1000)^{1 / 2}$, the price of the option, the lower (LL) and upper (UL) limits of the $95 \%$ confidence interval of $\mathrm{AV}$, and the percentage error $E(\mathrm{AV})$ related to $\mathrm{AV}$.

To the best of our knowledge, there is no result concerning the existence of locally risk minimizing hedging 
TABLE 2: Average hedging error of the one-touch option written on the CEV model.

\begin{tabular}{cccccccc}
\hline$k$ & Hedging strategies & AV & St. error & LL & UL & Price & $E(\mathrm{AV})$ \\
\hline 3 & 11 & 0.0449 & 0.0073 & 0.0305 & 0.0592 & 0.4803 & $9.34 \%$ \\
3 & 16 & 0.0446 & 0.0063 & 0.0323 & 0.0569 & 0.4804 & $9.28 \%$ \\
3 & 22 & 0.0441 & 0.0056 & 0.0332 & 0.0550 & 0.4804 \\
3 & 44 & 0.0431 & 0.0044 & 0.0345 & 0.0516 & 0.4803 & $8.18 \%$ \\
4 & 11 & 0.0213 & 0.0071 & 0.0017 & 0.0295 & 0.5062 \\
4 & 16 & 0.0203 & 0.0064 & 0.0078 & 0.0327 & 0.5060 \\
4 & 22 & 0.0167 & 0.0053 & 0.0062 & 0.0271 & 0.5061 & $4.00 \%$ \\
4 & 44 & 0.0158 & 0.0038 & 0.0084 & 0.0232 & 0.5057 \\
5 & 11 & 0.0067 & 0.0072 & -0.0074 & 0.0209 & 0.5205 & $3.12 \%$ \\
5 & 16 & 0.0056 & 0.0065 & -0.0186 & 0.0073 & 0.5196 & $1.30 \%$ \\
5 & 22 & 0.0050 & 0.0055 & -0.0057 & 0.0157 & 0.5187 & $0.97 \%$ \\
5 & 44 & 0.0044 & 0.0040 & -0.0034 & 0.0122 & 0.5204 & $0.85 \%$ \\
\hline
\end{tabular}

TABLE 3: Monte Carlo local risk minimization hedging strategy of a European put option with Heston model.

\begin{tabular}{ccccc}
\hline$k$ & Hedging & Standard error & Monte Carlo price & Standard error \\
\hline 3 & -0.4480 & $6.57 \times 10^{-4}$ & 10.417 & $5.00 \times 10^{-3}$ \\
4 & -0.4506 & $1.28 \times 10^{-3}$ & 10.422 & $3.35 \times 10^{-3}$ \\
5 & -0.4453 & $2.54 \times 10^{-3}$ & 10.409 & $2.75 \times 10^{-3}$ \\
\hline
\end{tabular}

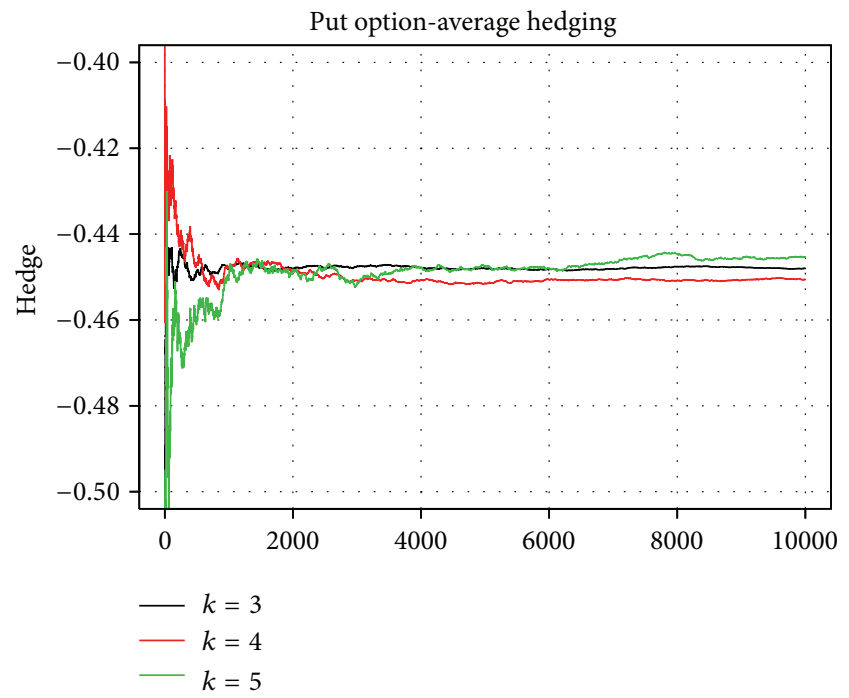

Figure 2: Monte Carlo local risk minimization hedging strategy of a European put option with Heston model.

strategies for one-touch options written on a Heston model with nonzero correlation. Nevertheless, as pointed out in Remark 20, it is expected that pure hedging strategies based on the generalized Föllmer-Schweizer decomposition mitigate very well the average hedging error. This is what we get in the simulation results. In Table 4 , we see that as $k$ increases, the percentage error $E(\mathrm{AV})$ decreases. For $k=3$, we also have a decrease in the standard error, but when $k=4$, the standard error is almost the same (with a small increase).

Mean Variance Hedging Strategy. Here we present the average hedging errors associated with one-touch options written on a Heston model with nonzero correlation under the mean variance methodology. Again, we simulate the average hedging error along the interval $[0,1]$ by using $k=3,4$ as discretization levels of the Brownian motions. We perform 22 and 44 hedging strategies in the period (which corresponds to one and two hedging strategies per day, resp.) with parameters $r=0, b=0.01, \kappa=3.63, \theta=0.04, \rho=-0.53$, $\sigma=0.3, Y_{0}=0.3$, and $S_{0}=100$. The barrier of the one-touch option (104) is 105. There are some quantities which are not related to the GKW decomposition that must be computed (see Remark 20). The quantity $\widetilde{\zeta}$ is not related to the GKW decomposition but it is described by Theorem 1.1 in Hobson [16] as follows. The process $\widetilde{\zeta}$ appearing in (5) and (6) is given by

$$
\widetilde{\zeta}_{t}=\widetilde{Z}_{0} \rho \sigma F(T-t)-\widetilde{Z}_{0} b ; \quad 0 \leq t \leq T,
$$

where $F$ is given by (see case 2 of Prop. 5.1 in [16])

$$
F(t)=\frac{C}{A} \tanh \left(A C t+\tanh ^{-1}\left(\frac{A B}{C}\right)\right)-B ; \quad 0 \leq t \leq T,
$$

with $A=\sqrt{\left|1-2 \rho^{2}\right| \sigma^{2}}, B=(\kappa+2 \rho \sigma b) / \sigma^{2}\left|1-2 \rho^{2}\right|$, and $C=\sqrt{|D|}$ where $D=2 b^{2}+(\kappa+2 \rho \sigma b)^{2} / \sigma^{2}\left(1-2 \rho^{2}\right)$. The initial condition $\widetilde{Z}_{0}$ is given by

$$
\widetilde{Z}_{0}=\frac{Y_{0}^{2}}{2} F(T)+\kappa \theta \int_{0}^{T} F(s) d s .
$$

The average hedging error results are summarized in Table 5. It reports the standard error (St. error) $=$ standard deviation of $\left\{\widehat{\gamma}_{\ell} ; 1 \leq \ell \leq M\right\} /$ (total number of scenarios $M=1000)^{1 / 2}$, the price of the option, the lower 
TABLE 4: Average hedging error with generalized Follmer-Schweizer decomposition: one-touch option with Heston model.

\begin{tabular}{cccccccc}
\hline$k$ & Hedging strategies & AV & St. error & LL & UL & Price & $E(\mathrm{AV})$ \\
\hline 3 & 22 & 0.0422 & 0.0084 & 0.0258 & 0.0586 & 0.7399 & $5.70 \%$ \\
3 & 44 & 0.0382 & 0.0067 & 0.0250 & 0.0515 & 0.7397 & $5.17 \%$ \\
4 & 22 & 0.0210 & 0.0080 & 0.0053 & 0.0366 & 0.7733 & $2.71 \%$ \\
4 & 44 & 0.0198 & 0.0082 & 0.0036 & 0.0360 & 0.7737 & $2.56 \%$ \\
\hline
\end{tabular}

(LL) and upper (UL) limits of the 95\% confidence interval of $\mathrm{AV}$, and the percentage error $E(\mathrm{AV})$ related to $\mathrm{AV}$. Compared to the local risk minimization methodology, the results show smaller percentage errors for $k=4$. Also, in all the cases, the results show smaller values of the standard errors which suggests the mean variance methodology provides more accurate values of the hedging strategy. Again, for a fixed value $k$, when the number of hedging strategies increases, the standard error decreases.

\section{Appendix}

This appendix provides a deeper understanding of the Monte Carlo algorithm proposed in this work when the representation $\left(\phi^{H, S}, \phi^{H, I}\right)$ in (43) admits additional integrability and path smoothness assumptions. We present stronger approximations which complement the asymptotic result given in Theorem 11. Uniform-type weak and strong pointwise approximations for $\theta^{H}$ are presented and they validate the numerical experiments in Tables 1 and 4 in Section 6. At first, we need some technical lemmas.

Lemma A.1. Suppose that $\phi^{H}=\left(\phi^{H, 1}, \ldots, \phi^{H, p}\right)$ is a $p$ dimensional progressive process such that $\mathbb{E}_{\sup _{0 \leq t \leq T}}\left\|\phi_{t}^{H}\right\|_{\mathbb{R}^{p}}^{2}<$ $\infty$. Then, the following identity holds:

$$
\begin{array}{r}
\Delta \delta^{k} X_{T_{1}^{k, j}}=\mathbb{E}\left[\int_{0}^{T_{1}^{k, j}} \phi_{s}^{H, j} d W_{s}^{(j)} \mid \mathscr{F}_{T_{1}^{k, j}}^{k}\right] \\
\text { a.s; } \quad j=1, \ldots, p ; \quad k \geq 1 .
\end{array}
$$

Proof. It is sufficient to prove for $p=2$ since the argument for $p>2$ easily follows from this case. Let $\mathscr{H}$ be the linear space constituted by the bounded $\mathbb{R}^{2}$-valued $\mathbb{F}$-progressive processes $\phi=\left(\phi^{1}, \phi^{2}\right)$ such that (A.1) holds with $X=X_{0}+$ $\int_{0}^{\int} \phi_{s}^{1} d W_{s}^{(1)}+\int_{0}^{\cdot} \phi_{s}^{2} d W_{s}^{(2)}$ where $X_{0} \in \mathscr{F}_{0}$. Let $\mathcal{U}$ be the class of stochastic intervals of the form $\llbracket S,+\infty \llbracket$ where $S$ is a $\mathbb{F}$ stopping time. We claim that $\phi=\left(\mathbb{1}_{\llbracket S,+\infty}, \mathbb{1}_{\mathbb{U} J,+\infty \pi}\right) \in \mathscr{H}$ for every $\mathbb{F}$-stopping times $S$ and $J$. In order to check (A.1) for such $\phi$, we only need to show for $j=1$ since the argument for $j=2$ is the same. With a slight abuse of notation, any subsigma-algebra of $\mathscr{F}_{T}$ of the form $\Omega_{1}^{*} \otimes \mathscr{G}$ will be denoted by $\mathscr{G}$ where $\Omega_{1}^{*}$ is the trivial sigma-algebra on the first copy $\Omega_{1}$.
At first, we split $\Omega=\bigcup_{n=1}^{\infty}\left\{T_{n}^{k}=T_{1}^{k, 1}\right\}$ and we make the argument on the sets $\left\{T_{n}^{k}=T_{1}^{k, 1}\right\}, n \geq 1$. In this case, we know that $\mathscr{F}_{T_{1}^{k, 1}}^{k}=\mathscr{F}_{T_{1}^{k, 1}}^{k, 1} \otimes \mathscr{F}_{T_{n-1}^{k, 2}}^{k, 2}$ a.s and

$$
\begin{aligned}
\Delta \delta^{k} X_{T_{1}^{k, j}}= & \Delta \delta^{k}\left(W_{T_{1}^{k, 1}}^{(1)}-W_{S}^{(1)}\right) \mathbb{1}_{\left\{S<T_{1}^{k, 1}\right\}} \\
& +\Delta \delta^{k}\left(W_{T_{1}^{k, 1}}^{(2)}-W_{J}^{(2)}\right) \mathbb{1}_{\left\{J<T_{1}^{k, 1}\right\}} .
\end{aligned}
$$

The independence between $W^{(1)}$ and $W^{(2)}$ and the independence of the Brownian motion increments yield

$$
\begin{aligned}
& \Delta \delta^{k}\left(W_{T_{1}^{k, 1}}^{(2)}-W_{J}^{(2)}\right) \\
& =\mathbb{E}\left(W_{T_{1}^{k, 1}}^{(2)}-W_{J}^{(2)} \mid \mathscr{F}_{T_{1}^{k, 1}}^{k}\right)-\mathbb{E}\left(W_{T_{1}^{k, 1}}^{(2)}-W_{J}^{(2)} \mid \mathscr{F}_{T_{n-1}^{k}}^{k}\right) \\
& =\mathbb{E}\left(W_{T_{1}^{k, 1}}^{(2)}-W_{J}^{(2)} \mid \mathscr{F}_{T_{1}^{k, 1}}^{k, 1} \otimes \mathscr{F}_{T_{n-1}^{k, 2}}^{k, 2}\right) \\
& =\mathbb{E}\left(W_{T_{1}^{k, 1}}^{(2)}-W_{J}^{(2)} \mid \sigma\left\{T_{1}^{k, 1}\right\} \otimes \mathscr{F}_{T_{n-1}^{k, 2}}^{k, 2}\right) \\
& =\mathbb{E}\left(W_{T_{1}^{k, 1}}^{(2)}-W_{J}^{(2)} \mid \mathscr{F}_{T_{n-1}^{k, 2}}^{k, 2}\right)=0 \text { a.s }
\end{aligned}
$$

on the set $\left\{T_{n-1}^{k} \leq J<T_{n}^{k}=T_{1}^{k, 1}\right\}$. We also have

$$
\begin{aligned}
\Delta \delta^{k} & \left(W_{T_{1}^{k, 1}}^{(2)}-W_{J}^{(2)}\right) \\
= & \mathbb{E}\left(W_{T_{1}^{k, 1}}^{(2)}-W_{J}^{(2)} \mid \mathscr{F}_{T_{1}^{k, 1}}^{k}\right)-\mathbb{E}\left(W_{T_{1}^{k, 1}}^{(2)}-W_{J}^{(2)} \mid \mathscr{F}_{T_{n-1}^{k}}^{k}\right) \\
= & \mathbb{E}\left(W_{T_{1}^{k, 1}}^{(2)}-W_{J}^{(2)} \mid \mathscr{F}_{T_{1}^{k, 1}}^{k, 1} \otimes \mathscr{F}_{T_{n-1}^{k, 2}}^{k, 2}\right) \\
& -\mathbb{E}\left(W_{T_{n-1}^{k}}^{(2)}-W_{J}^{(2)} \mid \mathscr{F}_{T_{n-1}^{k, 2}}^{k, 2}\right) \\
= & \mathbb{E}\left(W_{T_{1}^{k, 1}}^{(2)}-W_{J}^{(2)} \mid \sigma\left\{T_{1}^{k, 1}\right\} \otimes \mathscr{F}_{T_{n-1}^{k, 2}}^{k, 2}\right) \\
& -\mathbb{E}\left(W_{T_{n-1}^{(2)}}^{(2)}-W_{J}^{(2)} \mid \mathscr{F}_{T_{n-1}^{k, 2}}^{k, 2}\right) \\
= & \mathbb{E}\left(W_{T_{n-1}^{(2)}}^{(2)}-W_{J}^{(2)} \mid \mathscr{F}_{T_{n-1}^{k, 2}}^{k, 2}\right) \\
& -\mathbb{E}\left(W_{T_{n-1}^{(2)}}^{(2)}-W_{J}^{(2)} \mid \mathscr{F}_{T_{n-1}^{k, 2}}^{k, 2}\right)=0,
\end{aligned}
$$


TABLE 5: Average hedging error in the mean variance hedging methodology for one-touch option with Heston model.

\begin{tabular}{cccccccc}
\hline$k$ & Hedging strategies & AV & St. error & LL & UL & Price & $E($ AV) \\
\hline 3 & 22 & 0.0674 & 0.0052 & 0.0572 & 0.0777 & 0.7339 & $9.19 \%$ \\
3 & 44 & 0.0577 & 0.0044 & 0.0490 & 0.0663 & 0.7340 & $7.86 \%$ \\
4 & 22 & 0.0143 & 0.0056 & 0.0034 & 0.0252 & 0.7767 & $1.84 \%$ \\
4 & 44 & 0.0134 & 0.0038 & 0.0060 & 0.0209 & 0.7765 & $1.73 \%$ \\
\hline
\end{tabular}

on the set $\left\{J<T_{n-1}^{k}\right\}$. By construction $\mathscr{F}_{T_{1}^{k, 1}}^{k}=\mathscr{F}_{T_{1}^{k, 1}}^{k, 1} \otimes \mathscr{F}_{T_{n-1}^{k, 2}}^{k, 2}$ a.s and again the independence between $W^{(1)}$ and $W^{(2)}$ yields

$$
\begin{aligned}
\Delta \delta^{k} & \left(W_{T_{1}^{k, 1}}^{(1)}-W_{S}^{(1)}\right) \\
= & \mathbb{E}\left(W_{T_{1}^{k, 1}}^{(1)}-W_{S}^{(1)} \mid \mathscr{F}_{T_{1}^{k, 1}}^{k}\right)-\mathbb{E}\left(W_{T_{1}^{k, 1}}^{(1)}-W_{S}^{(1)} \mid \mathscr{F}_{T_{n-1}^{k}}^{k}\right) \\
= & \mathbb{E}\left(W_{T_{1}^{k, 1}}^{(1)}-W_{S}^{(1)} \mid \mathscr{F}_{T_{1}^{k, 1}}^{k}\right)
\end{aligned}
$$

on $\left\{T_{n-1}^{k} \leq S<T_{n}^{k}=T_{1}^{k, 1}\right\}$. Similarly,

$$
\begin{aligned}
\Delta \delta^{k} & \left(W_{T_{1}^{k, 1}}^{(1)}-W_{S}^{(1)}\right) \\
= & \mathbb{E}\left(W_{T_{1}^{k, 1}}^{(1)}-W_{S}^{(1)} \mid \mathscr{F}_{T_{1}^{k, 1}}^{k}\right)-\mathbb{E}\left(W_{T_{1}^{k, 1}}^{(1)}-W_{S}^{(1)} \mid \mathscr{F}_{T_{n-1}^{k}}^{k}\right) \\
= & \mathbb{E}\left(W_{T_{1}^{k, 1}}^{(1)}-W_{S}^{(1)} \mid \mathscr{F}_{T_{1}^{k, 1}}^{k}\right)-\mathbb{E}\left(W_{T_{n-1}^{k}}^{(1)}-W_{S}^{(1)} \mid \mathscr{F}_{T_{n-1}^{k}}^{k, 2}\right) \\
= & \mathbb{E}\left(W_{T_{1}^{k, 1}}^{(1)}-W_{S}^{(1)} \mid \mathscr{F}_{T_{1}^{k, 1}}^{k}\right)-\mathbb{E}\left(W_{T_{n-1}^{k}}^{(1)} \mid \mathscr{F}_{T_{n-1}^{k}}^{k, 2}\right) \\
& +\mathbb{E}\left(W_{S}^{(1)} \mid \mathscr{F}_{T_{n-1}^{k}}^{k, 2}\right) \\
= & \mathbb{E}\left(W_{T_{1}^{(1)}}^{(1)}-W_{S}^{(1)} \mid \mathscr{F}_{T_{1}^{k, 1}}^{k}\right)+\mathbb{E}\left(W_{S}^{(1)} \mid \mathscr{F}_{T_{n-1}^{k}}^{k, 2}\right)
\end{aligned}
$$

on $\left\{S<T_{n-1}^{k}\right\}$. By assumption $S$ is an $\mathbb{F}$-stopping time, where $\mathbb{F}$ is a product filtration. Hence, $\mathbb{E}\left(W_{S}^{(1)} \mid \underset{\mathcal{F}^{k, 2}}{T_{n-1}^{k}}\right)=0$ a.s on $\left\{S<T_{n-1}^{k}\right\}$.

Summing up the above identities, we will conclude

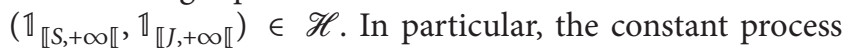
$(1,1) \in \mathscr{H}$ and if $\phi^{n}$ is a sequence in $\mathscr{H}$ such that $\phi^{n} \rightarrow \phi$ a.s Leb $\times \mathbb{Q}$ with $\phi$ bounded, then a routine application of Burkhölder inequality shows that $\phi \in \mathscr{H}$. Since $\mathscr{U}$ generates the optional sigma-algebra, we will apply the monotone class theorem and, by localization, we may conclude the proof.

Strong Convergence under Mild Regularity. In this section, we provide a pointwise strong convergence result for GKW projectors under rather weak path regularity conditions. Let us consider the stopping times

$$
\tau^{j}:=\inf \left\{t>0 ;\left|W_{t}^{(j)}\right|=1\right\} ; \quad j=1, \ldots, p
$$

and we set

$$
\psi^{H, j}(u):=\mathbb{E}\left|\phi_{\tau^{j} \mathcal{u}}^{H, j}-\phi_{0}^{H, j}\right|^{2}, \quad \text { for } u \geq 0, j=1, \ldots, p .
$$

Here, if $u$ satisfies $\tau^{j} u \geq T$, we set $\phi_{\tau^{j} u}^{H, j}:=\phi_{T}^{H, j}$ and for simplicity we assume that $\psi^{H, j}(0-)=0$.

Theorem A.2. If $H$ is a $\mathbb{Q}$-square-integrable contingent claim satisfying $(M)$ and there exists a representation $\phi^{H}=$ $\left(\phi^{H, 1}, \ldots, \phi^{H, p}\right)$ of $H$ such that $\phi^{H, j} \in B^{2}(\mathbb{F})$ for some $j \in$ $\{1, \ldots, p\}$ and the initial time $t=0$ is a Lebesgue point of $u \mapsto \psi^{H, j}(u)$, then

$$
\mathbf{D}^{k, j} X_{T_{1}^{k, j}} \longrightarrow \phi_{0}^{H, j} \quad \text { as } k \longrightarrow \infty .
$$

Proof. In the sequel, $C$ will be a constant which may differ from line to line and let us fix $j=1, \ldots, p$. For a given $k \geq 1$, it follows from Lemma A.1 that

$$
\begin{aligned}
\mathbb{D}^{k, j} & X_{T_{1}^{k, j}} \\
= & \frac{\mathbb{E}\left[\int_{0}^{T_{1}^{k, j}} \phi_{s}^{H, j} d W_{s}^{(j)} \mid \mathscr{F}_{\left.T_{1}^{k, j}\right]}^{k}\right.}{\Delta A_{T_{1}^{k, j}}^{k, j}} \\
= & \frac{\mathbb{E}\left[\int_{0}^{T_{1}^{k, j}}\left(\phi_{s}^{H, j}-\phi_{0}^{H, j}+\phi_{0}^{H, j}\right) d W_{s}^{(j)} \mid \mathscr{F}_{T_{1}^{k, j}}^{k}\right]}{\Delta A_{T_{1}^{k, j}}^{k, j}} \\
= & \frac{\mathbb{E}\left[\int_{0}^{T_{1}^{k, j}}\left(\phi_{s}^{H, j}-\phi_{0}^{H, j}\right) d W_{s}^{(j)} \mid \mathscr{F}_{T_{1}^{k, j}}^{k}\right]}{\Delta A_{T_{1}^{k, j}}^{k, j}} \\
& +\mathbb{E}\left[\phi_{0}^{H, j} \mid \mathscr{F}_{\left.T_{1}^{k, j}\right]}^{k}{ }^{k,}\right.
\end{aligned}
$$

We recall that $T_{1}^{k, j} \stackrel{\text { law }}{=} 2^{-2 k} \tau^{j}$ so that we will apply the Burkholder-Davis-Gundy and Cauchy-Schwartz inequalities 
Data: Maturity Time $T$, Discretization level $k$

Result: Vector of stopping times $\mathscr{T}^{k, j}$, family $\eta^{k, j}$, step processes $A^{k, j}$

(1) $\mathscr{T}^{k, j} \leftarrow$ Burq and Jones $(k, T) \quad$ Vector of $\mathbb{F}^{k, j}$-stopping times generated by

the algorithm described by Burq and Jones [8]

(2) $n \leftarrow \operatorname{Length}\left(\mathscr{T}^{k, j}\right) \quad n$ is the length of $\mathscr{T}^{k, j}$

(3) $\eta_{0}^{k, j} \leftarrow 0.0$

(4) $A_{0}^{k, j} \leftarrow 0.0$

(5) for $i \leftarrow 1$ to $n$ do

(6) $\quad \eta_{i}^{k, j} \leftarrow$ Sample one element from Bernoulli (0.5)

(7) $\quad A_{i}^{k, j} \leftarrow A_{i-1}^{k, j}+2^{-k} \eta_{i}^{k, j}$

Algorithm 1: Pseudocode of the stopping times $T^{k, j}$ and step processes $A^{k, j}$.

Data: Partitions $\mathscr{T}^{k, j}$, Families $\eta^{k, j}$ and Step Processes $A^{k, j}$ for $j=1, \ldots, p$, Discretization level $k$, Number of Stocks $d$

Result: Stock Prices $S^{k, i}, i=1, \ldots, d$

(1) $\mathscr{T}^{k} \leftarrow \operatorname{Merge}\left(\mathscr{T}^{k, j}\right) \quad$ Create the finest partition $\mathscr{T}^{k}$ from the partitions $\mathscr{T}^{k, j}$

(2) $n \leftarrow \operatorname{Length}\left(\mathscr{T}^{k}\right) \quad n$ is the length of $\mathscr{T}^{k}$

(3) for $i \leftarrow 1$ to $d$ do

(4) $\quad$ for $\ell \leftarrow 1$ to $n$ do

(5) $\quad S_{\ell}^{k, i} \leftarrow$ Itô-Taylor $\left(\mathscr{T}^{k}, \eta^{k, j}, A^{k, j} ; j=1, \ldots, p\right)$ Simulation of $S^{i}$ in the finest partition $\mathscr{T}^{k}$ using some Itô-Taylor approximation method

(6) $\widehat{H}^{k}=\Phi\left(S^{k, j} ; 1 \leq j \leq p\right) \quad$ Approximation of $H$ as a function of the simulated $S^{k, j}$

Algorithm 2: Pseudocode of the stock price processes $S^{i}, i=1, \ldots, d$.

together with a simple time change argument on the Brownian motion to get the following estimate:

$$
\begin{aligned}
& |\mathbb{E}| \frac{\mathbb{E}\left[\int_{0}^{T_{1}^{k, j}}\left(\phi_{s}^{H, j}-\phi_{0}^{H, j}\right) d W_{s}^{(j)} \mid \mathscr{F}_{T_{1}^{k, j}}^{k}\right]}{\Delta A_{T_{1}^{k, j}}^{k, j}} \mid \\
& \quad \leq 2^{k} \mathbb{E}\left|\int_{0}^{T_{1}^{k, j}}\left(\phi_{s}^{H, j}-\phi_{0}^{H, j}\right) d W_{s}^{(j)}\right| \\
& =2^{k} \mathbb{E}\left|\int_{0}^{2^{-2 k}}\left(\phi_{\tau^{j} s}^{H, j}-\phi_{0}^{H, j}\right) d W_{\tau^{j} s}^{(j)}\right| \\
& \leq\left. C 2^{k} \mathbb{E}\left|\int_{0}^{2^{-2 k}}\left(\phi_{\tau^{j} s}^{H, j}-\phi_{0}^{H, j}\right)^{2} \tau^{j} d s\right|\right|^{1 / 2} \\
& \quad \leq C\left(\mathbb{E} \tau^{j}\right)^{1 / 2}\left(\mathbb{E} \frac{1}{2^{-2 k}} \int_{0}^{2^{-2 k}}\left(\phi_{\tau^{j} u}^{H, j}-\phi_{0}^{H, j}\right)^{2} d u\right)^{1 / 2} \\
& \quad=C\left(\mathbb{E} \frac{1}{2^{-2 k}} \int_{0}^{2^{-2 k}}\left(\phi_{u \tau^{j}}^{H, j}-\phi_{0}^{H, j}\right)^{2} d u\right)^{1 / 2} \cdot
\end{aligned}
$$

strongly in $L^{1}$. Since $\mathbf{D}^{k, j} X_{T_{1}^{k, j}}=\mathbb{E}\left[\mathbb{D}^{k, j} X_{T_{1}^{k, j}}\right], k \geq 1$, we conclude the proof.

Remark A.3. At first glance, limit (A.9) stated in Theorem A.2 seems to be rather weak since it is not defined in terms of convergence of processes. However, from the purely computational point of view, we will construct a pointwise Monte Carlo simulation method of the GKW projectors in terms of $\mathbf{D}^{k, j} X_{T_{1}^{k, j}}$ given by (41). This substantially simplifies the algorithm introduced by Leão and Ohashi [27] for the unidimensional case under rather weak path regularity. 
Data: Partitions $\mathscr{T}^{k, j}$, Families $\eta^{k, j}$, Step Processes $A^{k, j}$, Maturity Time $T$, Discretization Level $k$, Number of Dimensions $p$, Number of Stocks $d$, Number of Simulations of the Conditional Expectation $L$, Number of Simulations of the Stochastic Derivative $M$

Result: Stochastic Derivatives $\mathbb{D}_{T_{1}^{k, j}}^{k, j} X, j=1, \ldots, d$, Hedging Strategy $\theta_{0}^{H}$

(1) $\mathscr{T} \leftarrow \operatorname{Merge}\left(\mathscr{T}^{k, j}\right) \quad$ Create the finest partition $\mathscr{T}^{k}$ from the partitions $\mathscr{T}^{k, j}$

(2) for $j \leftarrow 1$ to $p$ do

(3) $m_{j}^{k} \leftarrow$ which $\left(\mathscr{T}^{k}==T_{1}^{k, j}\right) \quad m_{j}^{k}$ is the position of $T_{1}^{k, j}$ in the finest partition $\mathscr{T}^{k}$

(4) for $i \leftarrow 1$ to $M$ do

(5) $\quad$ for $j \leftarrow 1$ to $p$ do

(6) $\quad \mathbb{D}^{k, j} X_{T_{1}^{k, j}} \leftarrow \frac{1}{2^{-k} \bar{\eta}_{1}^{k, j}}\left(\widehat{\mathbb{E}}\left[H \mid \mathscr{F}_{T_{1}^{k, j}}^{k}\right]-\widehat{\mathbb{E}}\left[H \mid \mathscr{F}_{\tau_{1}^{k, j}}^{k}\right]\right)$ Computations of $\mathbb{D}^{k, j} X_{T_{1}^{k, j}}$

(7) $\quad \phi_{0}^{H, S} \leftarrow$ concatenate $\left(\mathbb{D}^{k, 1} X_{T_{1}^{k, 1}}, \ldots, \mathbb{D}^{k, d} X_{T_{1}^{k, d}}\right)$ Vector of stochastic derivatives

(8) $\quad\left(\bar{\theta}_{0}^{H}\right)_{i} \leftarrow\left(\phi^{H, S}\right)^{\top}\left[\operatorname{diag}\left(S_{0}\right) \sigma_{0}\right]^{-1} \quad$ Create the vector of hedging strategy $\bar{\theta}_{0}^{H}$

(9) $\theta_{0}^{H} \leftarrow \operatorname{mean}(\bar{\theta}) \quad$ Hedging strategy $\theta_{0}^{H}$

Algorithm 3: Pseudocode of the stochastic derivative $\mathbb{D}^{k, j} X_{T_{1}^{k, j}}$ and the hedging strategy $\theta_{0}^{H}$.

Remark A.4. For each $j=1, \ldots, p$, let us define

$$
\psi^{H, j}\left(t_{0}, u\right):=\mathbb{E}\left|\phi_{t_{0}+\tau^{j} u}^{H, j}-\phi_{t_{0}}^{H, j}\right|^{2}, \quad \text { for } t_{0} \in[0, T], u \geq 0 \text {. }
$$

One can show by a standard shifting argument based on the Brownian motion strong Markov property that if there exists a representation $\phi^{H}$ such that $u \mapsto \psi^{H, j}\left(t_{0}, u\right)$ is cadlag for a given $t_{0}$, then one can recover in a pointwise manner in $L^{1}$ strong sense the $j$ th GKW projector for that $t_{0}$. We notice that if $\phi^{H, j}$ belongs to $B^{2}(\mathbb{F})$ and it has cadlag paths, then $u \mapsto \psi^{H, j}\left(t_{0}, u\right)$ is cadlag for each $t_{0}$, but the converse does not hold. Hence the assumption in Theorem A.2 is rather weak in the sense that it does not imply the existence of a cadlag version of $\phi^{H, j}$.

\section{Conflict of Interests}

The authors declare that there is no conflict of interests regarding the publication of this paper.

\section{Acknowledgments}

The authors would like to thank Bruno Dupire and Francesco Russo for stimulating discussions and several suggestions on the numerical algorithm proposed in this work. They also gratefully acknowledge the computational support from LNCC (Laboratório Nacional de Computação Científica, Brazil). The second author was supported by CNPq Grant 308742 .

\section{References}

[1] H. Föllmer and D. Sondermann, "Hedging of non-redundant contingent claims," in Contributions to Mathematical Economics, W. Hildenbrand and A. Mas-Colell, Eds., pp. 205-223, North Holland, Amsterdam, The Netherlands, 1986.
[2] M. Schweizer, "Option hedging for semimartingales," Stochastic Processes and Their Applications, vol. 37, no. 2, pp. 339-363, 1991.

[3] H. Föllmer and M. Schweizer, "Hedging of contingent claims under incomplete information," Applied Stochastic Analysis, Stochastic Monographs, vol. 5, pp. 389-414, 1991.

[4] D. Heath, E. Platen, and M. Schweizer, "A comparison of two quadratic approaches to hedging in incomplete markets," Mathematical Finance, vol. 11, no. 4, pp. 385-413, 2001.

[5] M. Schweizer, "A guided tour through quadratic hedging approaches," in Option Pricing, Interest Rates and Risk Management, E. Jouini, J. Cvitanic, and M. Musiela, Eds., pp. 538-574, Cambridge University Press, Cambridge, UK, 2001.

[6] L. Delong, Backward Stochastic Differential Equations with Jumps and Their Actuarial and Financial Applications, 2013.

[7] M. Schweizer, "On the minimal martingale measure and the möllmer-schweizer decomposition," Stochastic Analysis and Applications, vol. 13, no. 5, pp. 573-599, 1995.

[8] Z. A. Burq and O. D. Jones, "Simulation of brownian motion at first-passage times," Mathematics and Computers in Simulation, vol. 77, no. 1, pp. 64-71, 2008.

[9] T. Rheinlander and M. Schweizer, "On $L^{2}$-projections on a space of stochastic integrals," The Annals of Probability, vol. 25, no. 4, pp. 1810-1831, 1997.

[10] C. Gourieroux, J. P. Laurent, and H. Pham, "Mean-variance hedging and numéraire," Mathematical Finance, vol. 8, no. 3, pp. 179-200, 1998.

[11] M. Schweizer, "Approximation pricing and the varianceoptimal martingale measure," The Annals of Probability, vol. 24, no. 1, pp. 206-236, 1996.

[12] A. Černý and J. Kallsen, "On the structure of general meanvariance hedging strategies," The Annals of Probability, vol. 35, no. 4, pp. 1479-1531, 2007.

[13] J. Kallsen, J. Muhle-Karbe, and R. Vierthauer, "Asymptotic power utility-based pricing and hedging," Mathematics and Financial Economics, vol. 8, no. 1, pp. 1-28, 2014.

[14] D. Kramkov and M. Sirbu, "Sensitivity analysis of utilitybased prices and risk-tolerance wealth processes," The Annals of Applied Probability, vol. 16, no. 4, pp. 2140-2194, 2006.

[15] D. Kramkov and M. Sirbu, "Asymptotic analysis of utility-based hedging strategies for small number of contingent claims," 
Stochastic Processes and Their Applications, vol. 117, no. 11, pp. 1606-1620, 2007.

[16] D. Hobson, "Stochastic volatility models, correlation, and the $q$ optimal measure," Mathematical Finance, vol. 14, no. 4, pp. 537556, 2004.

[17] F. Biagini, P. Guasoni, and M. Pratelli, "Mean-variance hedging for stochastic volatility models," Mathematical Finance, vol. 10, no. 2, pp. 109-123, 2000.

[18] S. L. Heston, "A closed-form solution for options with stochastic volatility with applications to bond and currency options," Review of Financial Studies, vol. 6, no. 2, pp. 327-343, 1993.

[19] J. Kallsen and R. Vierthauer, "Quadratic hedging in affine stochastic volatility models," Review of Derivatives Research, vol. 12, no. 1, pp. 3-27, 2009.

[20] A. Černý and J. Kallsen, "Mean-variance hedging and optimal investment in Heston's model with correlation," Mathematical Finance, vol. 18, no. 3, pp. 473-492, 2008.

[21] E. Gobet and C. Labart, "Error expansion for the discretization of backward stochastic differential equations," Stochastic Processes and Their Applications, vol. 117, no. 7, pp. 803-829, 2007.

[22] J. Zhang, "A numerical scheme for BSDEs," The Annals of Applied Probability, vol. 14, no. 1, pp. 459-488, 2004.

[23] E. Gobet and A. Makhlouf, " $\mathrm{L}_{2}$-time regularity of BSDEs with irregular terminal functions," Stochastic Processes and Their Applications, vol. 120, no. 7, pp. 1105-1132, 2010.

[24] P. Briand and C. Labart, "Simulation of BSDEs by Wiener chaos expansion," The Annals of Applied Probability, vol. 24, no. 3, pp. 1129-1171, 2014.

[25] E. Gobet and P. Turkedjiev, "Approximation of backward stochastic differential equations using malliavin weights and least-squares regression," To appear in Bernoulli.

[26] E. Gobet and P. Turkedjiev, "Linear regression MDP scheme for discrete backward stochastic differential equations under general conditions," preprint.

[27] D. Leão and A. Ohashi, "Weak approximations for Wiener functionals," The Annals of Applied Probability, vol. 23, no. 4, pp. 1660-1691, 2013.

[28] B. Dupire, "Functional Itô calculus," Bloomberg Porfolio Research Paper 04, 2009.

[29] R. Cont and D.-A. Fournié, "Functional Itô calculus and stochastic integral representation of martingales," The Annals of Probability, vol. 41, no. 1, pp. 109-133, 2013.

[30] A. Ohashi, D. Leão, and A. B. Simas, "Weak functional Itô calculus and applications," http://arxiv.org/abs/1408.1423.

[31] C. Dellacherie and P.-A. Meyer, Probabilities and Potential, Volume B, North-Holland Publishing, Amsterdam, The Netherlands, 1982.

[32] C. Dellacherie, P.-A. Meyer, and M. Yor, "Sur certaines proprietes des espaces de Banach $\mathrm{H}^{1}$ et $\mathrm{BMO}$," in Séminaire de Probabilités XII: Université de Strasbourg 1976/77, vol. 649, pp. 98-113, 1978.

[33] S.-W. He, C.-K. Wang, and J.-A. Yan, Semimartingale Theory and Stochastic Calculus, Taylor \& Francis, 1992.

[34] G. Bernis, E. Gobet, and A. Kohatsu-Higa, "Monte Carlo evaluation of Greeks for multidimensional barrier and lookback options," Mathematical Finance, vol. 13, no. 1, pp. 99-113, 2003. 


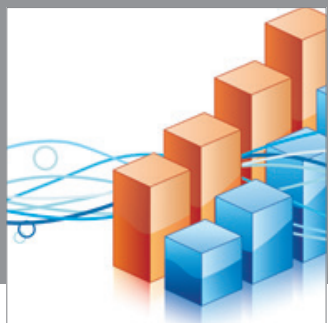

Advances in

Operations Research

mansans

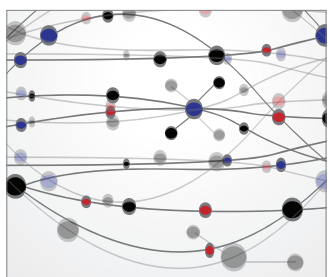

The Scientific World Journal
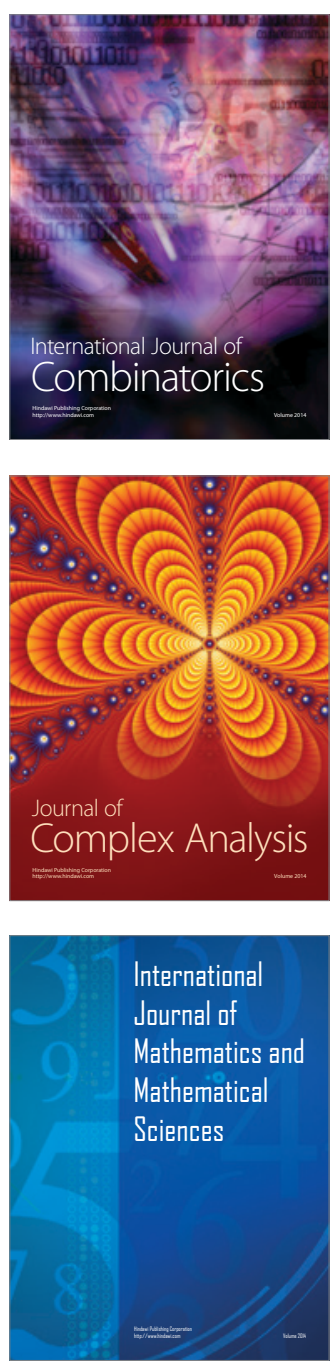
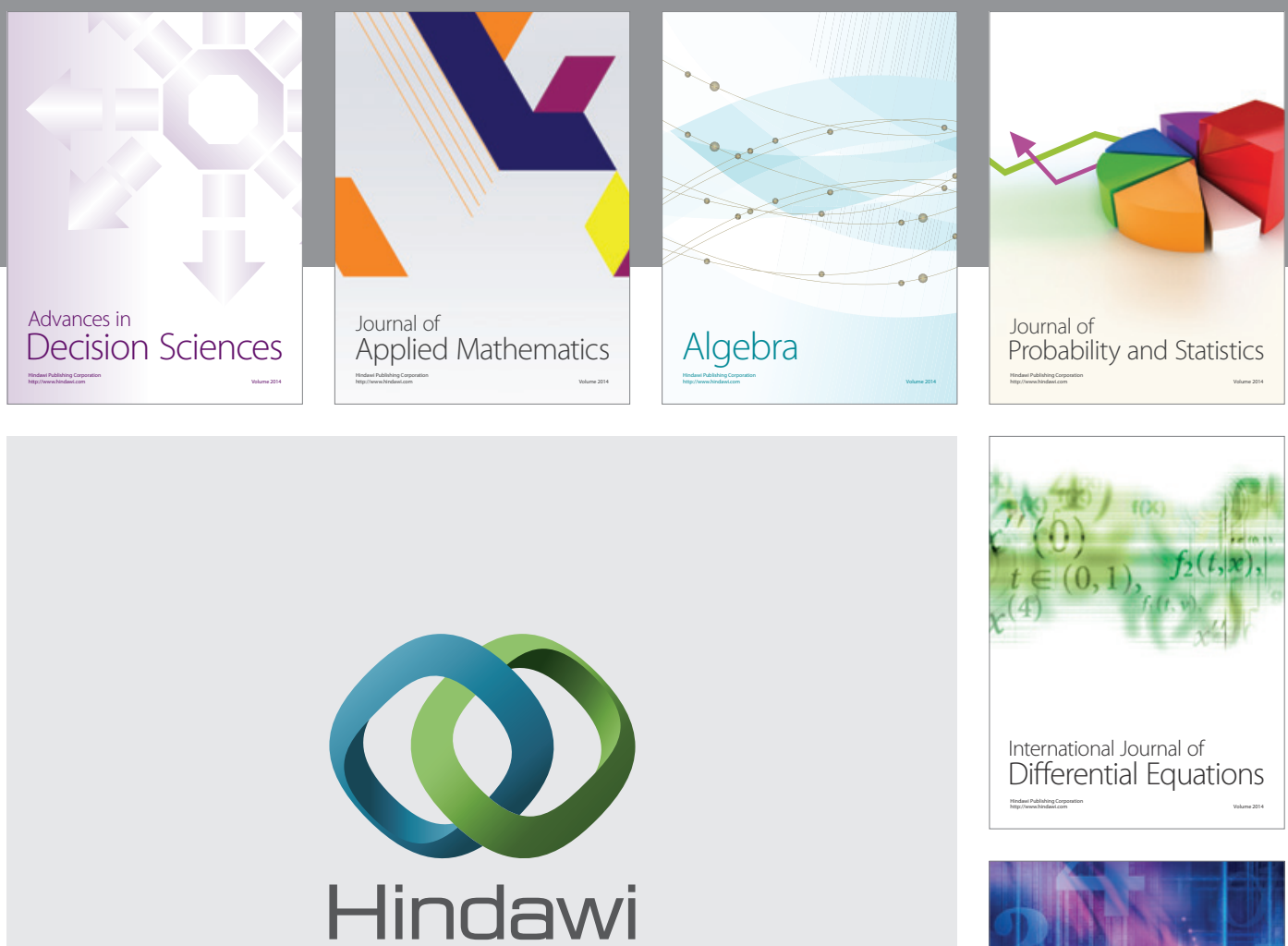

Submit your manuscripts at http://www.hindawi.com
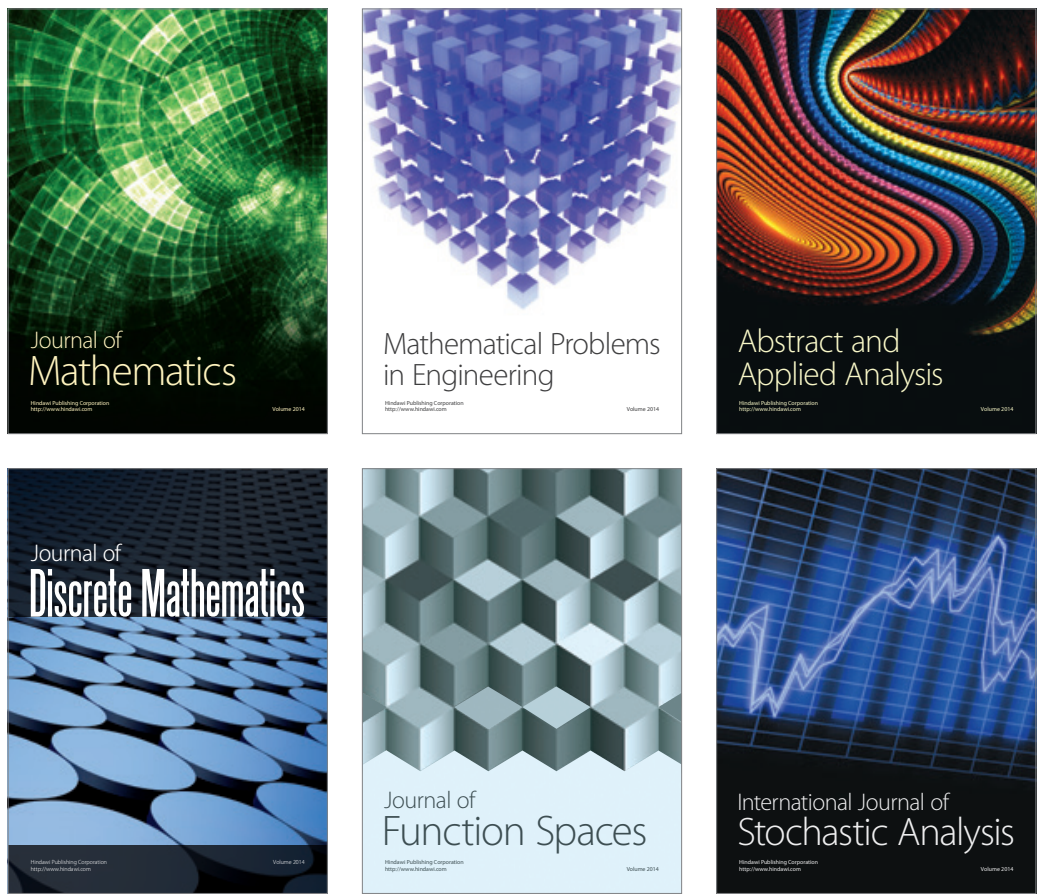

Journal of

Function Spaces

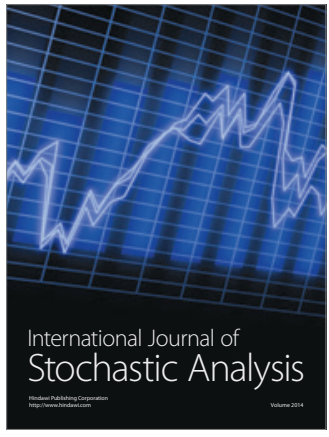

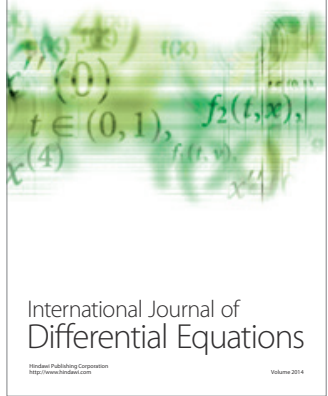
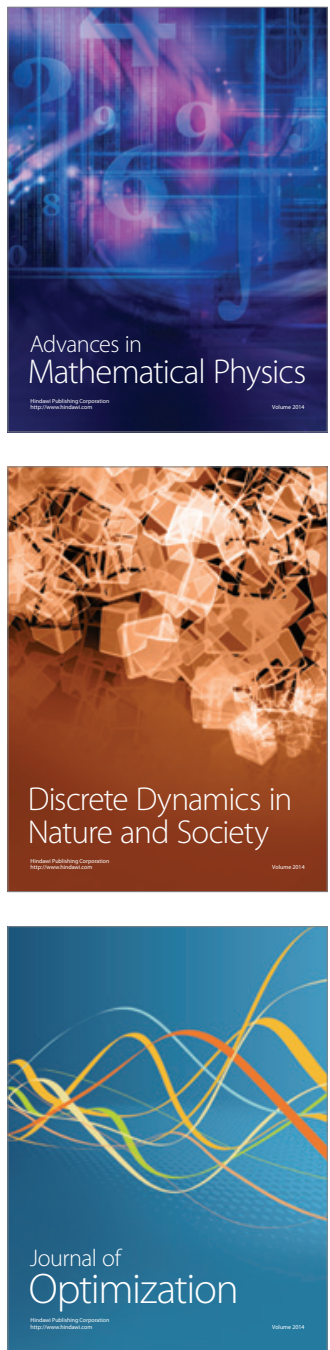